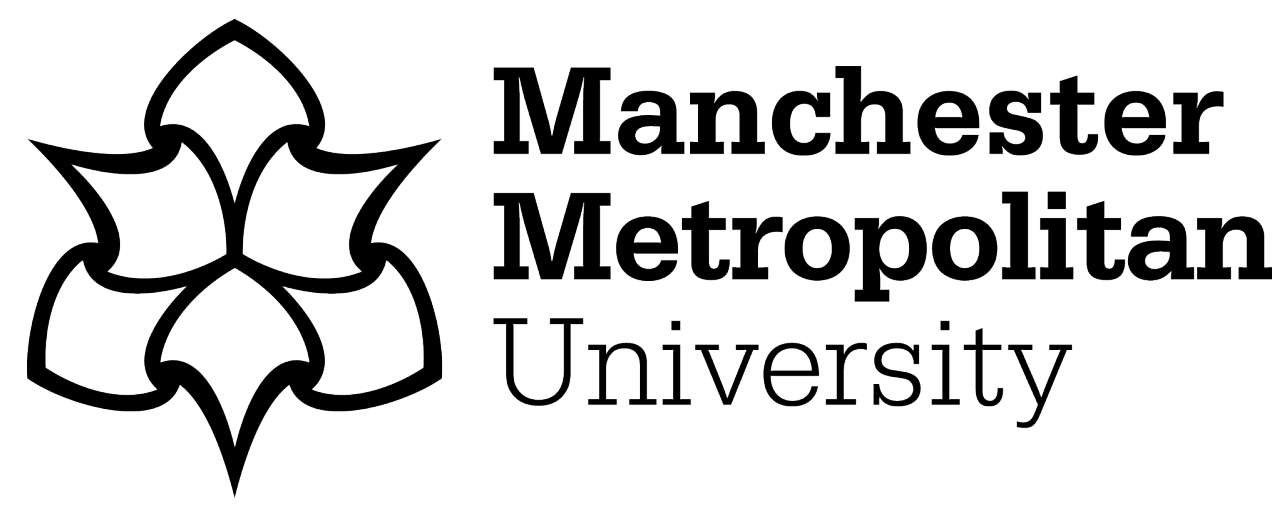

Zhen, Li, Bashir, Ali Kashif ORCID logoORCID: https://orcid.org/0000-00017595-2522, Yu, Keping, Al-Otaibi, Yasser D, Foh, Chuan Heng and Xiao, Pei (2021) Energy-Efficient Random Access for LEO Satellite-Assisted 6G Internet of Remote Things. IEEE Internet of Things Journal, 8 (7). pp. 51145128.

Downloaded from: https://e-space.mmu.ac.uk/626668/ Version: Accepted Version

Publisher: Institute of Electrical and Electronics Engineers (IEEE)

DOI: https://doi.org/10.1109/jiot.2020.3030856

Please cite the published version 


\title{
Energy-Efficient Random Access for LEO Satellite-Assisted 6G Internet of Remote Things
}

\author{
Li Zhen, Member, IEEE, Ali Kashif Bashir, Senior Member, IEEE, Keping Yu, Member, IEEE, \\ Yasser D. Al-Otaibi, Chuan Heng Foh, Senior Member, IEEE, and Pei Xiao, Senior Member, IEEE
}

\begin{abstract}
Satellite communication system is expected to play a vital role for realizing various remote internet of things (IoT) applications in 6G vision. Due to unique characteristics of satellite environment, one of the main challenges in this system is to accommodate massive random access $(R A)$ requests of IoT devices while minimizing their energy consumptions. In this paper, we focus on the reliable design and detection of RA preamble to effectively enhance the access efficiency in highdynamic low-earth-orbit (LEO) scenarios. To avoid additional signaling overhead and detection process, a long preamble sequence is constructed by concatenating the conjugated and circularly shifted replicas of a single root Zadoff-Chu (ZC) sequence in RA procedure. Moreover, we propose a novel impulse-like timing metric based on length-alterable differential cross-correlation (LDCC), that is immune to carrier frequency offset (CFO) and capable of mitigating the impact of noise on timing estimation. Statistical analysis of the proposed metric reveals that increasing correlation length can obviously promote the output signal-to-noise power ratio, and the first-path detection threshold is independent of noise statistics. Simulation results in different LEO scenarios validate the robustness of the proposed method to severe channel distortion, and show that our method can achieve significant performance enhancement in terms of timing estimation accuracy, success probability of first access, and mean normalized access energy, compared with the existing RA methods.
\end{abstract}

Index Terms-6G, satellite communication, internet of remote things, random access, energy efficiency.

\section{INTRODUCTION}

This work was supported in part by the Natural Science Foundation of China (NSFC) under Grants 61901370 and 62001381, in part by the Special Research Project of Education Department of Shaanxi Province under Grant 19JK0794, in part by the Open Fund of the Shaanxi Key Laboratory of Information Communication Network and Security under Grant ICNS201801, in part by the Science and Technology Innovation Team of Shaanxi Province for Broadband Wireless and Application under Grant 2017KCT-30-02, and in part by the Japan Society for the Promotion of Science (JSPS) Grants-in-Aid for Scientific Research (KAKENHI) under Grant JP18K18044. (Corresponding author: Keping $\mathrm{Yu}$ ).

L. Zhen is with the Shaanxi Key Laboratory of Information Communication Network and Security, Xi'an University of Posts and Telecommunications, Xi'an, China (e-mail: lzhen@xupt.edu.cn).

A. K. Bashir is with the Department of Computing and Mathematics, Manchester Metropolitan University, U.K., and School of Information and Communication Engineering, University of Electronics Science and Technology of China (UESTC), Chengdu, China (e-mail: Dr.alikashif.b@ieee.org).

$\mathrm{K}$. Yu is with the Global Information and Telecommunication Institute, Waseda University, Tokyo, Japan, and Shenzhen Boyi Technology Co., Ltd, Shenzhen, China (e-mail: keping.yu@aoni.waseda.jp).

Y. D. Al-Otaibi is with the Department of Information Systems, Faculty of Computing and Information Technology in Rabigh, King Abdulaziz University, Jeddah 21589, Saudi Arabia (e-mail: yalotaibi@kau.edu.sa).

C. H. Foh and P. Xiao are with the Institute for Communication Systems, University of Surrey, Guildford, U.K. (e-mails: c.foh@surrey.ac.uk, p.xiao@surrey.ac.uk).

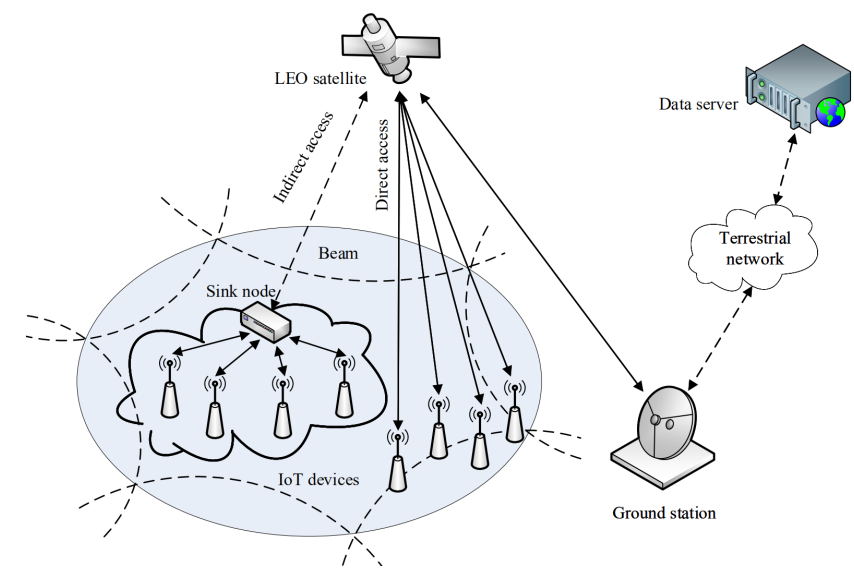

Fig. 1. System architecture of LEO satellite-assisted internet of remote things.

W ITH the ongoing deployment of 5th-generation (5G) cellular systems, research focus toward the nextgeneration wireless communications has been put on the agenda by both academics and industries [1]-[3]. Driven by the explosive growth of smart devices and data traffic, 6th generation $(6 \mathrm{G})$ system is aimed at building a largedimensional and autonomous global network able to support massive and unlimited wireless connectivity [4]. In this regard, the emerging wide-area wireless communication technologies for internet of things (IoT) scenarios, such as narrow-band IoT, LoRa, and unmanned aerial vehicle (UAV) communication, are far from satisfying the seamless coverage requirement of ubiquitous devices in 6G-enabled global IoT. As shown in Fig. 1 , for many IoT applications, devices are usually distributed over an extremely wide area or located in remote areas lacking of terrestrial infrastructure, which makes low-earthorbit (LEO) satellite communication a promising solution in realizing remote data interaction given its global coverage [5].

To perform uplink data transmission, each IoT device needs to initiate a random access (RA) procedure for network connection with the base station. When a device attempts to access the base station, it randomly selects one of the available preambles from the given preamble set to transmit on a RA time slot. Upon successful detection of the preamble sequences, the base station allocates the physical uplink shared channel (PUSCH) resources ${ }^{1}$ and replies the RA response messages to the corresponding devices in the

\footnotetext{
${ }^{1}$ In general, PUSCH resources are mainly used for uplink user data transmission, and thus only a small amount of $\mathrm{PUSCH}$ resources are reserved for the RA procedure.
} 
subsequent step of RA procedure. However, different from the terrestrial communication scenarios, a massive number of devices exist in the satellite beam with a wider coverage, they usually initiate simultaneous access requests to the loadlimited satellite receiver for emergency alarm and status report [6]. In this case, severe preamble collision and congestion will unavoidably occur, leading to frequently access retries and extra time spent on the RA procedure for the collided devices, which causes additional energy consumptions to their already limited energy supplies. It is therefore imperative to study how to improve the access efficiency of IoT devices to minimize the energy consumption in satellite communication scenarios.

\section{A. Relative Work}

There have been a limited works focusing on designing energy-efficient RA schemes [7]-[13], in which the aforementioned RA overload problem, caused by the deficient contention resource of preambles and PUSCHs, was regarded as the main driver of energy consumption. The solutions to this problem can be categorized into preamble collision mitigation, access class control, resource allocation, as well as power control, all of which, nevertheless, are dependent on the premise of a successful preamble detection at the first step of RA procedure. However, this fundamental premise may not be guaranteed by taking into account the unique characteristics of LEO satellite environment, such as wide beam coverage, long propagation distance, and rapid relative movement between satellite and ground, which bring great challenges in effective design and detection of RA preamble.

With the massive footprint of a satellite beam, the length of preamble must be sufficiently extended to absorb the significantly increased propagation delay, compared to that in terrestrial scenarios. Such that, the durations of cyclic prefix (CP) and guard time (GT) should be lengthened for the intersymbol interference (ISI) avoidance among the adjacent subframes, and in the meanwhile, a longer preamble sequence is also required to fulfill the requirements of coverage performance. With the long propagation distance resulting in a high path loss, the preamble detection process should work reliably in the conditions of low received signal-to-noise ratios (SNRs). Besides, the rapid relative movement between LEO satellite and IoT device signifies a high-dynamic communication environment, and thus can give rise to the considerable Doppler shift and channel dispersion, which requires the preamble detection method be capable of having the robustness to large carrier frequency offset (CFO) and multi-path effect. Given the above, special attention should be paid to the reasonable design and reliable detection of RA preamble for LEO satellite-based IoT communications.

With regard to this critical issue, some preamble design and detection methods presented in long term evolution (LTE)satellite systems [14], [15] could be considered for reference. In [16], He et al. leveraged the preamble design principles in LTE [17] to estimate the differential round trip delay and then compensated it before preamble transmission, such that both the durations of CP and GT can be greatly reduced. Even though, the sequence duration has to be lengthened in large scale to provide a sufficient beam coverage, which results in a small sub-carrier interval of RA preamble more vulnerable to large CFO. By considering the system compatibility, Li et al. [18] proposed to cascade the same terrestrial Zadoff-Chu (ZC) sequences to constitute the preamble sequence. Although the fractional timing advance (TA) could be effectively achieved, an extra preamble is required to transmit for determining the integral TA. In [19], Zhen et al. further designed a long preamble sequence based on multiple short sequences generated by cyclic shifting a root $\mathrm{ZC}$ sequence with large sub-carrier spacing, so that both the fractional and integral TA of each user can be determined by performing once power delay profile (PDP) calculation, which notably improves both the access efficiency and performance. However, the fractional and integral TA values need to be estimated separately, and the residual CFO will also lead to a decline of timing estimation accuracy. In addition, the methods in [20] and [21] presented to exploit the conjugate-symmetric $\mathrm{ZC}$ sequences to eliminate the impact of integral CFO on timing estimation, but both methods suffered from the obvious performance degradation in the presence of fractional CFO. Recently, 3GPP had offered some potential solutions in preamble design for $5 \mathrm{G}$ integrated satellite network [22], wherein Gold/m-sequence was considered as one of the candidate preamble sequences. Nevertheless, the additional processing procedures are indispensable, and thus a high computational complexity will be introduced that may be difficult to sustain for the load-limited satellite receiver.

\section{B. Contribution}

It is foreseen from the features of $6 \mathrm{G}$-enabled IoT communications [23]-[25] that, RA procedure is one of the most frequently used and energy consuming operations in devices. As the foremost step in RA procedure, an appropriate design and effective detection of RA preamble can remarkably enhance the success probability of first access, and is consequently conducive to reducing both the energy consumption and average access delay, so as to improve the access efficiency for massive IoT devices. However, the assumption of perfect preamble detection in the existing energy-efficient RA schemes might be too optimistic by considering the complicated and changeable LEO satellite environments, which make the previously presented preamble design and detection methods for pute satellite line-of-sight (LOS) scenarios no longer applicable. In consequence, a more reliable one with the robustness to severe channel distortion is required for energy-efficient RA procedure, especially in LEO satellite-based IoT. Inspired by this fact, we are dedicated to developing a new RA preamble and the corresponding timing detection method with the aims of achieving the resistivity to $\mathrm{CFO}$, the mitigation of noise, as well as the insensitivity to channel dispersion. The main contributions of this work are as follows:

1) We propose an enhanced single-root cascaded long sequence (E-SCLS). By only replacing the first subsequence of SCLS [19] to its conjugate form, it absolutely inherits the advantage of SCLS in supporting coverage extension, and more importantly, can achieve one-step TA estimation by directly detecting the elaborate conjugate 
root sequence without additional signaling overhead and detection process.

2) We present a novel timing metric according to the construction features of E-SCLS. Specifically, by jointly utilizing the starting two sub-sequences of E-SCLS, a length-alterable differential cross-correlation (LDCC) with an impulse-like shape, instead of the conventional periodical correlation, is designed as the correlation function, which not only is immune to CFO, but also can take advantage of the whole correlation products between the two sequences.

3) We investigate the statistical properties of the proposed timing metric for different classifications of timing positions within the detection window. It is found that our timing metric with a flexibly adjusted correlation length is capable of effectively restraining the adverse impact of noise on timing estimation, and the detection threshold used to accurately track the first arrival path can be easily determined with no need for noise variance estimation.

4) We validate the feasibility of the proposed timing estimation method in different LEO satellite channels, from the aspects of timing mean square error, success probability of first access, and mean normalized access energy. We further analyze and compare the characteristics of our methods in [19] and in this paper, and give suggestions on choosing a more appropriate method according to the actual communication scenario parameters, e.g. user location and elevation angle.

The remainder of this paper is organized as follows. We describe the RA preamble design in satellite environments and formulate the received signal model in Section II. Section III elaborates the proposed E-SCLS, the timing metric based on LDCC as well as its statistical analysis. Numerical results and discussions are presented in Section IV. Finally, Section VI concludes the paper.

\section{BACKGROUND AND SYSTEM DESCRIPTION}

\section{A. RA Preamble Design in Satellite Environments}

RA preamble is used for user identification and TA estimation, which usually has an uniform time-domain structure in terrestrial communication systems. Because of the long propagation delay and large beam radius, the maximum differential round trip delay can reach to several milliseconds in satellite scenarios, which can be hundred times longer than that in terrestrial scenarios. Such that, the preamble duration should be greatly enlarged to cope with the time uncertainty, causing an obvious increment in detection complexity. Besides, the more noteworthy problem is the high sensitivity of RA preamble to Doppler and other sources of CFO. Since the subcarrier spacing is the reciprocal of sequence duration, it would be substantially compressed in regard to a long sequence, leading to a significant timing performance degradation in the presence of large CFOs. Therefore, it is necessary to design a more appropriate preamble format for the load-limited satellite communication systems.

For this purpose, we had proposed an enhanced RA preamble format in our previous work [19], wherein the long sequence is constructed by concatenating a string of short sequences immune to CFO. By effectively designing the subcarrier spacing of short sequence in terms of the maximum Doppler shift related to the user mobility ${ }^{2}$, the adverse impact of CFO on preamble detection can be mitigated in advance. In this case, the cascaded sequence not only can satisfy the coverage requirements within the beam, but also achieves the CFO-mitigation capability. Specifically, let us denote the durations of long sequence and short sequence as $T_{L S}$ and $T_{S S}$, respectively, $K=\left\lfloor T_{L S} / T_{S S}\right\rfloor$ time-domain short sequences are utilized to construct the long sequence, while $\mathrm{ZC}$ sequences are chosen to generate the short sequence defined as $S_{n}=\exp [-j \pi u n(n+1) / N]$ for $0 \leq n \leq N-1$, where $u \in\{1, \ldots, N-1\}$ is the root index and $N$ denotes the sequence length. Without loss of generality, the transmitted signal model of the long sequence can be formulated as

$$
x_{n}=\left\{\begin{array}{l}
S_{n}^{1}, \quad 1 \leq n \leq N \\
S_{n-N}^{2}, \quad N+1 \leq n \leq 2 N \\
\vdots \\
S_{n-(K-1) N}^{K}, \quad(K-1) N+1 \leq n \leq K N
\end{array}\right.
$$

where $x_{n}$ denotes the $n$th element of the long sequence for $1 \leq n \leq K N$, and $S^{k}$ is the $k$ th short sequence for $1 \leq k \leq \bar{K}$. By considering the fact that $\mathrm{ZC}$ sequences obtained by cyclically shifting a single root sequence are mutually orthogonal [17], we put forward to adopt the same root sequence with a fixed cyclic shifts to constitute each short sequence, i.e. $S_{n}^{k}=S_{n}\left(\left(n+(k-1) N_{C S}\right) \bmod N\right)$, where $N_{C S}=\lfloor N / K\rfloor$ is the cyclic shift offset. In this case, the timing detection for the long preamble only needs to be performed in one shot by merging all the short sequences achieved from the same root sequence. In consequence, utilization of such preamble sequence, named as single-root cascaded long sequence (SCLS), not only can avoid the non-orthogonal interference introduced by using different root sequences, but also is conducive to reduce detection complexity for the loadlimited satellite receiver.

\section{B. Received Signal Model}

In the beam coverage area, when a machine node attempts to access the satellite, it will select one of the available preamble sequences to transmit on a periodical RA slot, and then initiates the RA procedure. After passing through a satellite multi-path fading channel with the relative path delays $\left\{\tau_{l}, 0 \leq l \leq L-1\right\}$ and the path gains $\left\{h_{l}, 0 \leq l \leq L-1\right\}$, the received signal sample has the following form of

$$
r_{n}=e^{j 2 \pi \varepsilon n / K N} \sum_{l=0}^{L-1} h_{l} x_{n-\tau_{l}-\tau}+w_{n},
$$

where $L, \tau$, and $\varepsilon$ represent the number of arriving paths, the first-path delay, and the CFO normalized by the RA subcarrier interval, respectively, while $w_{n}$ is a complex additive

\footnotetext{
${ }^{2}$ It is notable that, once the orbit parameters of an LEO satellite are determined, the Doppler shift corresponding to the movement of the satellite can be basically estimated and compensated for. Hence, we considered CFO to be the Doppler shift that caused mainly by the movement of user in [19].
} 
white Gaussian noise (AWGN) sample with mean zero and variance $\sigma_{w}^{2}$.

Then, the satellite receiver performs a reversed procedure corresponding to the RA preamble transmission. Specifically, $K$ sub-sequences with length of $N$ in time domain can be obtained by a decascading process with $\mathrm{CP}$ removal, equispaced division, discrete Fourier transform (DFT), sub-carrier demapping, and inverse DFT. Subsequently, the receiver carries out the timing detection based on the obtained $K$ sub-sequences to identify different users and estimate their individual values of TA. By means of the estimated TA, each user could adjust the transmission time in advance to ensure the sent signals of multi-users arriving at the satellite simultaneously, such that the uplink synchronization is finally achieved.

In [19], a timing detection scheme based on SCLS had also been presented in terms of PDP calculation, peak search and TA estimation. By the utilization of piecewise accumulation of the received sub-sequences, the PDP calculation can be performed only once to capture all the correlation peaks in the concentrated detection window, such that both the fractional and integral TA can be determined according to the unique feature of its cyclic shift set, which effectively reduces the computational complexity of the satellite receiver. Although satisfactory timing estimation performance is achieved in the satellite LOS channel, this method may experience a significant performance degradation when applied to the satellite multi-path channel with severe channel distortion, which is mainly caused by the following reasons:

1) Absence of $\mathrm{CFO}$ resistance: $\mathrm{ZC}$ sequence is susceptible to $\mathrm{CFO}$ in OFDM systems with small sub-carrier interval. Since there is no effective CFO resistance in the correlationbased PDP calculation, large CFOs due to the high Doppler shift and the instability of oscillator make the correlation peak shift or decrease, and further affect the TA estimation result.

2) Vulnerable to strong noise: When the received SNR is relatively low, the detection threshold needs to be correspondingly increased for mitigating the adverse impact of noise, such that some weak correlation peaks can not be successfully extracted, resulting in a TA estimation error.

3) Sensitive to channel dispersion: In case where the scattering path dominates, the correlation peak corresponding to the scattering path is likely to exceed the detection threshold and thus the wrong peak position will be captured, which also reduces the multi-peak joint TA estimation accuracy ${ }^{3}$.

From these points of view, the previously presented RA preamble design and detection method may have great difficulties to be applied to the complicated and changeable LEO satellite channel, and consequently a more robust one with significant performance improvement is required for realizing reliable satellite-based IoT.

\footnotetext{
${ }^{3}$ By using the piecewise cumulative detection in [19], multiple peaks with different locations can be obtained in once periodical correlation operation, which can be further used to estimate the fractional and integral values of TA, respectively. However, peak shifts will inevitably occur if the scattering path dominates, leading to the decline of TA estimation accuracy.
}

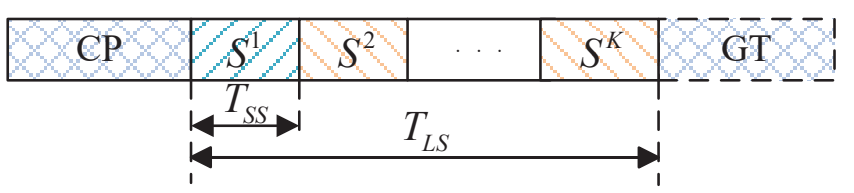

Fig. 2. The structure of the proposed E-SCLS.

\section{Robust Design of RA Preamble and Detection METHOD}

In this section, we concentrate on the design of the RA preamble and detection method with the aim of achieving the robustness to severe satellite channel conditions. To be specific, we first consider constructing a more reasonable preamble sequence, and further present the corresponding timing detection scheme for TA estimation.

\section{A. An Enhanced Preamble Sequence E-SCLS}

SCLS comprises of a root ZC sequence and its cyclic shifted replicas. As aforementioned, the detection performance degradation for the long sequence may occur in the presence of large CFOs, strong noise, and serious channel dispersion. This undesirable result is mainly caused by the non-uniqueness of captured correlation peaks since each sub-sequence in SCLS employs the same root index. Moreover, it can be found that the value of TA is actually corresponding to the propagation delay of the first sub-sequence in the received RA preamble. Therefore, a feasible solution is to improve the sequence component of SCLS to make the first sub-sequence obviously different from the others, so as to extract a unique and correct correlation peak in the PDP detection window.

Since the periodical correlation result between different root sequences is not zero, the introduction of extra root sequences leads to inevitable non-orthogonal interference during the preamble detection procedure [26]. Motivated by this fact, we present an enhanced single-root cascaded long sequence (E-SCLS) as shown in Fig. 2, wherein only the first subsequence of SCLS, i.e. the short root ZC sequence, is replaced with its conjugate form, and can be expressed as $S_{n}^{1}=$ $\exp [j \pi u n(n+1) / N]$ for $0 \leq n \leq N-1$. In this case, all the sub-sequences of the proposed E-SCLS are still constructed based on a single root sequence, and thus the non-orthogonal interference can be effectively suppressed. More importantly, the adoption of the proposed E-SCLS is of great help to further enhance computational efficiency during the preamble detection procedure, in comparison with those in [18] and [19]. This is because the value of TA can be completely determined by effectively detecting the elaborate conjugate root sequence, without performing two correlation operations to estimate the fractional and integral TA, respectively.

\section{B. Proposed Timing Metric Design Based on LDCC}

According to the structure characteristics of the proposed E-SCLS, TA estimation is actually to determine the time index corresponding to the start of the received conjugate root sequence. Towards this end, the corresponding timing detection method for E-SCLS should be able to achieve a 
unique and distinct correlation peak at the desired timing position, which is easily captured in the detection window and could be further utilized for correct timing estimation. However, the high sensitivity of $\mathrm{ZC}$ sequence to large $\mathrm{CFO}$ makes this target greatly challenging [27], [28], especially for the LEO satellite scenario with high Doppler shift and unpredictable oscillator error.

There are two major solutions to cope with the adverse influence of large CFO: 1) Setting larger sub-carrier spacing for physical RA channel (PRACH) based on the maximum Doppler shift [19]. Since ZC sequence is capable of resisting to CFO less than half a sub-carrier interval, if the sub-carrier interval is set to be greater than or equal to two times of maximum Doppler shift, the impact of CFO on timing detection can be restrained in advance. 2) Using the conjugate-symmetric ZC sequences [20], [21]. By combining a root ZC sequence with its conjugate sequence to construct the preamble sequence, the integral CFO can be effectively eliminated because the effects of integral CFO on the two correlation peaks are just the opposite. Unlike existing solutions that adopt conventional periodical correlation as timing metric, our solution takes a further step by designing a novel timing metric with the robustness to CFO.

As described in [29], [30], differential cross-correlation (DCC), that is immune to CFO, has been widely adopted for the timing detection of pseudo-noise (PN) sequence. In the following, we first utilize this function as the timing metric for E-SCLS, and study its timing estimation performance in RA preamble detection. As aforementioned, as long as the initial position of the first short ZC sequence is found in the detection window, the differential round-trip delay of the transmitted preamble can be determined, that is, the value of TA. Let $\mathbf{S}=\left\{S_{1}, \ldots, S_{N}\right\}$ denote the first short ZC sequence of E-SCLS, and each element of local differential vector is generated by conjugate multiplication of the adjacent elements in $\mathbf{S}$, i.e. $S_{n}{ }^{*} S_{n+1}$ for $n=1, \ldots, N-1$, where $(\cdot)^{*}$ denotes the complex conjugate operator. In the same way, the received differential vector can be constructed by the received sequence $\mathbf{r}^{\mathbf{d}}=\left\{r_{1}^{d}, \ldots, r_{N}^{d}\right\}$ at time index $d$, where the $n$th element $r_{n}^{d}$ is also expressed as $r_{d+n}$. By performing the cross-correlation between the two reconstructed sequences, the timing metric based on DCC can be given by

$$
T(d)=\frac{1}{N-1} \sum_{n=1}^{N-1}\left(S_{n}^{*} S_{n+1}\right)^{*}\left(r_{d+n}^{*} r_{d+n+1}\right) .
$$

We now analyze the influence of CFO on the correlation peak of DCC. At the correct timing position $d_{c}$, a full-sequence pattern match occurs, thus we have $d_{c}=\tau+\tau_{l}$ for the $l$ th arriving path. In this case, substituting (2) to (3) and neglecting the noise term, the correlation peak $T\left(d_{c}\right)$ can be expressed as

$$
\begin{aligned}
T\left(d_{c}\right) & =\frac{1}{N-1} \sum_{n=1}^{N-1}\left|h_{l}\right|^{2}\left(S_{n}^{*} S_{n+1}\right)^{*}\left(S_{n}^{*} S_{n+1} e^{j 2 \pi \varepsilon / K N}\right) \\
& =e^{j 2 \pi \varepsilon / K N} \frac{\left|h_{l}\right|^{2}}{N-1} \sum_{n=1}^{N-1}\left|S_{n}\right|^{2}\left|S_{n+1}\right|^{2} .
\end{aligned}
$$

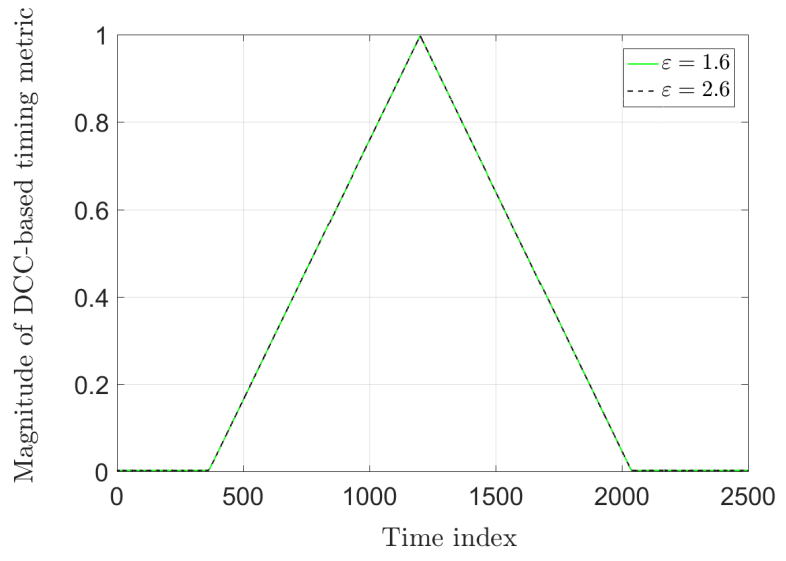

Fig. 3. An illustration of DCC-based timing metric in a noiseless channel.

It is found from (4) that the impact of CFO on $T\left(d_{c}\right)$ becomes a constant exponential factor, i.e. $e^{j 2 \pi \varepsilon / K N}$. By performing the modular operation on $T\left(d_{c}\right)$, this factor is equal to one, which is independent of the desired peak value at the correct time index. Thus, the adverse effect of CFO on preamble detection can be eliminated.

To clarify, we further depict the magnitude of the DCCbased timing metric under a noiseless LOS channel for different normalized CFOs in Fig. 3, where the parameters $K, N, h_{l}$, and $\tau$ are set as $3,839,1,1200$, respectively. It can be observed that, even if CFO is larger than one subcarrier interval, the magnitude of timing metric still obtains its maximum value at the correct timing position, and meanwhile, is not affected by the varying CFOs. Nevertheless, its timing curve has a triangle shape, and the peak difference between adjacent positions is great small. This is due to the specific characteristics of $\mathrm{ZC}$ sequence that the differential vector generated by one single $\mathrm{ZC}$ sequence will no longer possess perfect zero auto-correlation property, and thus its timing metric is not a impulse-like shape as expected. It is foreseeable that the timing estimation of single ZC sequence based DCC is highly susceptible to noise and multi-path effect, and will suffer from serious performance degradation when applied to the poor satellite environment with low received SNRs and severe channel dispersion. Based on the above analysis, we focus attention on designing a more robust timing detection function with a notably distinguishable correlation peak, which not only can resist to large CFO, but also is capable of mitigation of noise.

Knowing that excellent correlation property will not maintain for the differential sequence built from one single $\mathrm{ZC}$ sequence, we try to jointly use the two $\mathrm{ZC}$ sequences, i.e. the first and second short sequences of the long preamble, to generate the local differential matrix. Let $\mathbf{S}^{\mathbf{1}}=\left\{S_{1}^{1}, \ldots, S_{N}^{1}\right\}$ and $\mathbf{S}^{\mathbf{2}}=\left\{S_{1}^{2}, \ldots, S_{N}^{2}\right\}$ be the first and second short sequences, which represent the conjugate and cyclic shift forms of a root ZC sequence, respectively. By leveraging diverse differential intervals between the two sequences, we can construct the local joint differential matrix with $(N-1) \times(N-1)$ dimen- 


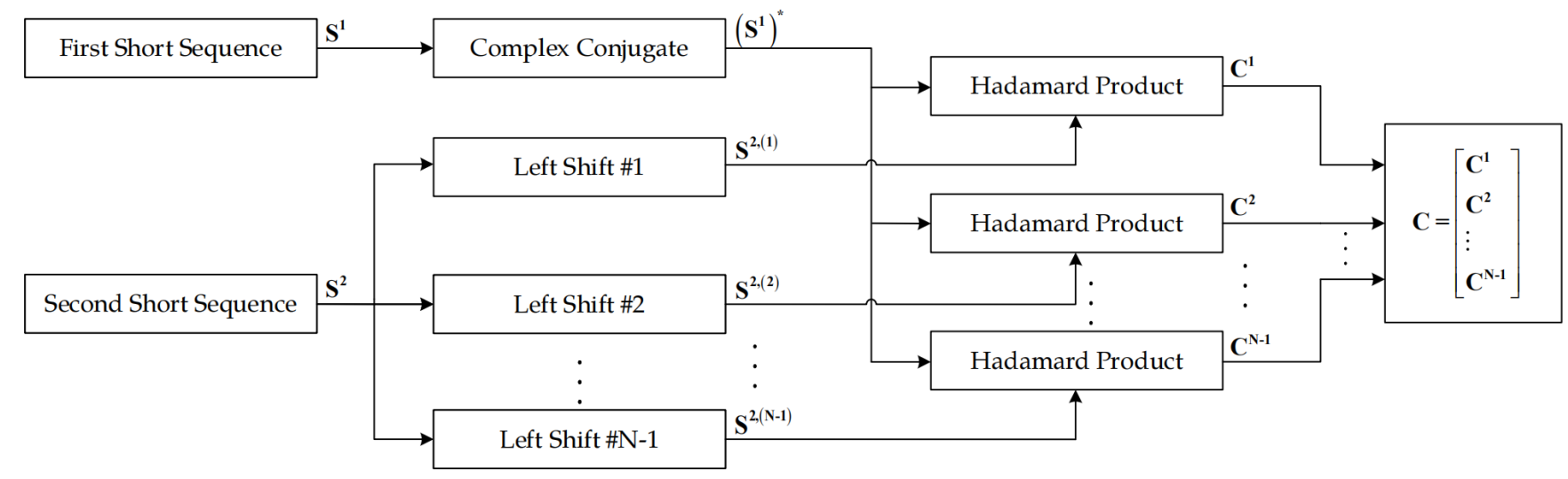

Fig. 4. The forming process of the local joint differential matrix.

sions as follows:

$$
\mathbf{C}=\left[\begin{array}{ccccc}
\left(S_{1}^{1}\right)^{*} S_{2}^{2} & \left(S_{2}^{1}\right)^{*} S_{3}^{2} & \cdots & \left(S_{N-2}^{1}\right)^{*} S_{N-1}^{2} & \left(S_{N-1}^{1}\right)^{*} S_{N}^{2} \\
\left(S_{1}^{1}\right)^{*} S_{3}^{2} & \left(S_{2}^{1}\right)^{*} S_{4}^{2} & \cdots & \left(S_{N-2}^{1}\right)^{*} S_{N}^{2} & 0 \\
\vdots & \vdots & . \cdot & . \cdot & \vdots \\
\left(S_{1}^{1}\right)^{*} S_{N-1}^{2} & \left(S_{2}^{1}\right)^{*} S_{N}^{2} \cdot \cdot \cdot & . \cdot & 0 \\
\left(S_{1}^{1}\right)^{*} S_{N}^{2} & 0 & \cdots & 0 & 0
\end{array}\right]
$$

To be specific, we exhibit the detailed generation diagram of $\mathbf{C}$ in Fig. 4. Note that the $m$ th row vector in $\mathbf{C}$ is obtained by $\mathbf{C}^{\mathbf{m}}=\left(\mathbf{S}^{\mathbf{1}}\right)^{*} \circ \mathbf{S}^{\mathbf{2},(\mathbf{m})}$, where $\mathbf{S}^{\mathbf{2},(\mathbf{m})}$ represents the vector achieved by left shifting $\mathbf{S}^{2}$ an amount of $m$, and o denotes the Hadamard product. In addition, the total number of non-zero elements for correlation reaches to be $N(N-1) / 2$, which is far greater than that in (3), i.e. $(N-1)$. As demonstrated later, this remarkable increment in the number of available correlation terms will lead to a better timing detection performance.

Next, $\mathbf{r}^{\mathbf{1}, \mathbf{d}}=\left\{r_{1}^{1, d}, \ldots, r_{N}^{1, d}\right\}$ is denoted as the first received short sequence at time index $d$, and $\mathbf{r}^{\mathbf{2}, \mathbf{d}}=\left\{r_{1}^{2, d}, \ldots, r_{N}^{2, d}\right\}$ is the second received short sequence next to $\mathbf{r}^{\mathbf{1}, \mathbf{d}}$. The received joint differential matrix $\mathbf{R}^{\mathbf{d}}$ is still constructed corresponding to the form of local matrix in (5), i.e.

$$
\mathbf{R}^{d}=\left[\begin{array}{ccccc}
\left(r_{1}^{1, d}\right)^{*} r_{2}^{2, d} & \left(r_{2}^{1, d}\right)^{*} r_{3}^{2, d} & \ldots & \left(r_{N-2}^{1, d}\right)^{*} r_{N-1}^{2, d} & \left(r_{N-1}^{1, d}\right)^{*} r_{N}^{2, d} \\
\left(r_{1}^{1, d}\right)^{*} r_{3}^{2, d} & \left(r_{2}^{1, d}\right)^{*} r_{4}^{2, d} & \ldots & \left(r_{N-2}^{1, d}\right)^{*} r_{N}^{2, d} & 0 \\
\vdots & \vdots & . \cdot & . \cdot & \vdots \\
\left(r_{1}^{1, d}\right)^{*} r_{N-1}^{2, d}\left(r_{2}^{1, d}\right)^{*} r_{N}^{2, d} & . \cdot & . \cdot & 0 \\
\left(r_{1}^{1, d}\right)^{*} r_{N}^{2, d} & 0 & \ldots & 0 & 0
\end{array}\right]
$$

By performing the cross-correlation between the corresponding elements in $\mathbf{C}$ and $\mathbf{R}^{\mathbf{d}}$, the two sequences-aided correlation function at time index $d$ can be expressed as

$$
P(d)=\sum_{m=1}^{M}\left|P_{m}(d)\right|=\sum_{m=1}^{M}\left|\sum_{n=1}^{N-m} C_{m, n}^{*} R_{m, n}^{d}\right|,
$$

where $P_{m}(d)$ represents the correlation results of the $m$ th rows in $\mathbf{C}$ and $\mathbf{R}^{\mathbf{d}}, M$ is the number of rows involved in correlation and $1 \leq M \leq N-1$. Note that, in (7), the impact of $\mathrm{CFO}$ on $P_{m}(d)$ remains a constant exponential factor, i.e. $e^{j 2 \pi(N+m) \varepsilon / K N}$, which can also be removed by performing the modular operation on the correlation function. That is to say, the proposed correlation function inherits the outstanding feature of DCC, and consequently is immune to CFO. On the other hand, it is observed that the number of available correlation terms in (7) is $(N / 2)$ times of that in (3), which means the utilization of two preamble sequences can effectively increase the correlation length. Because the preamble sequence is uncorrelated with noise samples, the difference between the correlation results in the absence and presence of two sequences (i.e. at the wrong and correct timing positions) will be significantly greater than that in the case of using one sequence for correlation. Therefore, increasing the correlation length can remarkably suppress the effect of noise on timing estimation (This point is further verified by the mathematical analysis in the following section). In order to achieve the best tradeoff between performance and computational complexity, the design parameter $M$ can be flexibly adjusted according to an excepted timing performance, thus we name the two sequences-aided correlation function as length-alterable DCC (LDCC).

For the existing RA preamble detection methods, the length of preamble sequence is always taken as the normalization factor. However, their timing metrics are more sensitive to the inevitable power fluctuation, and thus may not be able to carry out the accurate TA estimation under low SNRs. With the aim of eliminating the adverse effect of power fluctuation, the normalization function is designed as shown in (8), such that the detection threshold can be set in a fixed range from 0 to 1 .

$$
R(d)=\sum_{m=1}^{M} \sum_{n=1}^{N-m}\left|R_{m, n}^{d}\right|
$$

Finally, by combining the correlation function in (7) and the normalization function in (8), the proposed two sequencesaided timing metric based on LDCC can be obtained as

$$
T_{p}(d)=P(d) / R(d), \quad 0 \leq d \leq K N-1
$$

Fig. 5 shows the curves of the proposed timing metric based on LDCC in (a) noiseless channel and (b) AWGN channel at $\mathrm{SNR}=-5 \mathrm{~dB}$. Here, the available number of correlation rows $M$ is 4 , and the other simulation parameters are as same values in Fig. 3 for fair comparison. It is obvious in Fig. 5(a) that, the 

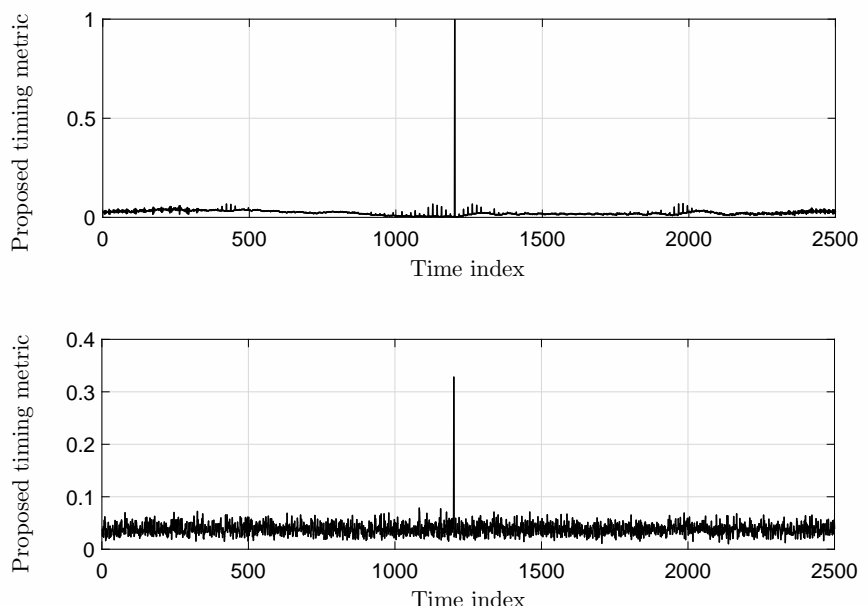

Fig. 5. Timing metric based on LDCC in (a) noiseless channel and (b) AWGN channel.

proposed metric has an impulse-like shape compared to DCC, and obtains its maximum value of 1 at the correct time index, which verifies the correctness of the designed normalization function. In Fig. 5(b), the proposed timing metric is affected by low SNR, but it can still achieve a notably distinguishable correlation peak, which further demonstrates its feasibility in the satellite LOS channel with strong main path. Moreover, the value of TA can be directly determined though one-step detection without additional signaling overhead [18] and detection procedure [19], and consequently the access efficiency is significantly improved.

\section{Statistical Analysis of The Proposed Timing Metric}

In order to reveal the superiority of proposed timing metric, we now analyze its statistical characteristics under the satellite multi-path fading channel in this section. According to the different classifications of timing positions within the detection window, we respectively derive the probability distributions of the proposed timing metric at the correct timing position and a wrong timing position, namely, in the presence and absence of two preamble sequences.

1) Correct timing position: For each arriving path, the corresponding correct timing position is denoted as $d_{c} \in$ $\left\{\tau+\tau_{l}, 0 \leq l \leq L-1\right\}$. In this case, the transmitted preamble is precisely received, and the two received preamble sequences can be respectively expressed as

$$
r_{n}^{1, d_{c}}=e^{j 2 \pi \varepsilon\left(n+d_{c}\right) / K N} \sum_{l=0}^{L-1} h_{l} S_{n}^{1}+w_{n+d_{c}}^{1}
$$

and

$$
r_{n}^{2, d_{c}}=e^{j 2 \pi \varepsilon\left(n+N+d_{c}\right) / K N} \sum_{l=0}^{L-1} h_{l} S_{n}^{2}+w_{n+d_{c}}^{2} .
$$

Submitting (10) and (11) to (7), the correlation result for $m$ th rows $P_{m}\left(d_{c}\right)$ can be rewritten as

$$
\begin{aligned}
& P_{m}\left(d_{c}\right) \\
& =\sum_{n=1}^{N-m} S_{n}^{1}\left(S_{n+m}^{2}\right)^{*}\left(e^{j 2 \pi \varepsilon\left(n+d_{c}\right) / K N} h_{l} S_{n}^{1}+w_{n+d_{c}}^{1}\right)^{*} \\
& \times\left(e^{j 2 \pi \varepsilon\left(n+N+d_{c}+m\right) / K N} h_{l} S_{n+m}^{2}+w_{n+d_{c}+m}^{2}\right),
\end{aligned}
$$

where the $l$ th relative path delay is $\tau_{l}=d_{c}-\tau$. Taking account of the zero auto-correlation property of ZC sequence, we ignore the influence of other paths with relative path delay of $\tau_{l} \neq d_{c}-\tau$ in (12).

Since the phase rotation of noise does not affect its probability distribution, $P_{m}\left(d_{c}\right)$ can be further given by

$$
\begin{aligned}
& P_{m}\left(d_{c}\right)= \\
& e^{\frac{j 2 \pi \varepsilon(N+m)}{K N}}\left\{\sum_{n=1}^{N-m}\left(\begin{array}{l}
\left|h_{l}\right|^{2}\left|S_{n}^{1}\right|^{2}\left|S_{n+m}^{2}\right|^{2} \\
+\left(h_{l}\right)^{*} S_{n}^{1}\left|S_{n+m}^{2}\right|^{2}\left(w_{n+d_{c}}^{1}\right)^{*} \\
+h_{l}\left|S_{n}^{1}\right|^{2}\left(S_{n+m}^{2}\right)^{*} w_{n+d_{c}+m}^{2} \\
+S_{n}^{1}\left(S_{n+m}^{2}\right)^{*}\left(w_{n+d_{c}}^{1}\right)^{*} w_{n+d_{c}+m}^{2}
\end{array}\right)\right\}
\end{aligned}
$$

Then we respectively define the four terms on the right side of the above equation as follows:

$$
\begin{gathered}
A_{m}=\sum_{n=1}^{N-m}\left|h_{l}\right|^{2}\left|S_{n}^{1}\right|^{2}\left|S_{n+m}^{2}\right|^{2} \\
Y_{m}=\sum_{n=1}^{N-m}\left(h_{l}\right)^{*} S_{n}^{1}\left|S_{n+m}^{2}\right|^{2}\left(w_{n+d_{c}}^{1}\right)^{*} \\
Z_{m}=\sum_{n=1}^{N-m} h_{l}\left|S_{n}^{1}\right|^{2}\left(S_{n+m}^{2}\right)^{*} w_{n+d_{c}+m}^{2} \\
W_{m}=\sum_{n=1}^{N-m} S_{n}^{1}\left(S_{n+m}^{2}\right)^{*}\left(w_{n+d_{c}}^{1}\right)^{*} w_{n+d_{c}+m}^{2}
\end{gathered}
$$

As a result, the correlation function $P_{m}\left(d_{c}\right)$ can be finally determined as

$$
P\left(d_{c}\right)=\sum_{m=1}^{M}\left|P_{m}\left(d_{c}\right)\right|=\sum_{m=1}^{M}\left|A_{m}+Y_{m}+Z_{m}+W_{m}\right| .
$$

We know from (13) that there exists an exponential factor, i.e. $e^{j 2 \pi \varepsilon(N+m) / K N}$, introduced by $\mathrm{CFO}$, which could be directly removed by the modular operation of $P_{m}\left(d_{c}\right)$ in (18). Besides, the normalization function that accumulate the absolute value of element in $\mathbf{R}^{\mathbf{d}_{\mathbf{c}}}$ can also eliminate the impact of CFO. As a result, the proposed timing metric is robust to CFO.

As aforementioned, the increment of correlation length can effectively mitigate the influence of noise for the proposed timing metric, and consequently results in a considerably better timing estimation performance. Note that, in (18), $Y_{m}, Z_{m}$, and $W_{m}$ are all affected by noise. In the following, we shall derive the statistical properties of these three random variables to understand the noise-mitigation capability of the proposed timing metric.

Let us consider $Y_{m}$ in (15) first. It easily follows that $Y_{m}$ contains $(N-m)$ mutually independent noise terms. For 
each noise term, thanks to the constant amplitude character of ZC sequence that $\left|S_{n}^{1}\right|^{2}=\left|S_{n+m}^{2}\right|^{2}=1$, and by considering the fact that phase rotation will not change the probability distribution of noise, each term in $Y_{m}$ obeys the complex Gaussian distribution, its mean and variance are 0 and $\left|h_{l}\right|^{2} \sigma_{w}^{2}$, respectively. Such that, $Y_{m}$ could be considered as the summation of independently and identically distributed (i.i.d.) complex random variables, and thus is a complex Gaussian random variable with mean 0 and variance $\left|h_{l}\right|^{2}(N-m) \sigma_{w}^{2}$.

For the variable $Z_{m}$ in (16), similarly, and can obtain that $Z_{m}$ follows the same probability distribution as $Y_{m}$. However, the time indexes of noise terms in $Y_{m}$ and $Z_{m}$ are different, thus $w_{n+d_{c}}^{1}$ and $w_{n+d_{c}+m}^{2}$ are mutually independent. Further, while $Y_{m}$ and $Z_{m}$ are independent of each other, their sum $Y_{m}+Z_{m}$ is still a complex Gaussian random variable with mean 0 and variance $2\left|h_{l}\right|^{2}(N-m) \sigma_{w}^{2}$.

We now consider the variable $W_{m}$ in (17). As the product of $S_{n}^{1}$ and $\left(S_{n+m}^{2}\right)^{*}$ is an exponential factor, each term in $W_{m}$ can be regarded as the phase rotation of $\left(w_{n+d_{c}}^{1}\right)^{*} w_{n+d_{c}+m}^{2}$. Since $w_{n+d_{c}}^{1}$ and $w_{n+d_{c}+m}^{2}$ are i.i.d. complex Gaussian random variables, it can be known from [31] that their product approximately follows complex Gaussian distribution with mean 0 and variance $\sigma_{w}^{2}$. Consequently, $W_{m}$ is also a complex Gaussian distribution random variable, and its variance is $(N-m) \sigma_{w}^{2}$.

For convenience, we assume $B_{m}$ to be the noise component in $P_{m}$, that is $B_{m}=Y_{m}+Z_{m}+W_{m}$. It is clear that $B_{m}$ can be seen as the sum of two complex Gaussian random variables, i.e. $Y_{m}+Z_{m}$ and $W_{m}$. Note that $Y_{m}+Z_{m}$ and $W_{m}$ are uncorrelated, since the covariance between them is calculated as

$$
\operatorname{Cov}\left\{Y_{m}+Z_{m}, W_{m}\right\}=E\left\{\left(Y_{m}+Z_{m}\right)^{*} W_{m}\right\}=0,
$$

where $\operatorname{Cov}\{\cdot\}$ and $E\{\cdot\}$ represent covariance operation and expectation operation, respectively. Because the uncorrelated complex Gaussian random variables are independent of each other, $Y_{m}+Z_{m}$ and $W_{m}$ are mutually independent. Accordingly, $B_{m}$ subjects to a complex Gaussian distribution, and its mean and variance are respectively the sum of those of $Y_{m}+Z_{m}$ and $W_{m}$, specifically, we can achieve

$$
E\left\{B_{m}\right\}=0
$$

and

$$
\operatorname{Var}\left\{B_{m}\right\}=\left(2\left|h_{l}\right|^{2}+1\right)(N-m) \sigma_{w}^{2},
$$

where $\operatorname{Var}\{\cdot\}$ denotes the variance operation.

To illustrate the noise-mitigation capability of the proposed timing metric, we further compared the output ratio $P R_{\text {out }}$ of the signal to noise power for different correlation lengths. Here, we shall focus on the output power ratio of the correlation function, since the normalization function does not affect the analysis of $P R_{\text {out }}$.

If the number of available correlation rows is 1 , i.e. $M=1$, $\left.P R_{\text {out }}\right|_{M=1}$ is given by

$$
\left.P R_{\text {out }}\right|_{M=1}=E\left\{\left|A_{1}\right|^{2}\right\} / E\left\{\left|B_{1}\right|^{2}\right\} \text {. }
$$

When $M=M_{0}$, and $2 \leq M_{0} \leq N-1,\left.P R_{\text {out }}\right|_{M=M_{0}}$ is expressed as

$$
\left.P R_{\text {out }}\right|_{M=M_{0}}=E\left\{\left[\sum_{m=1}^{M_{0}}\left|A_{m}\right|\right]^{2}\right\} / E\left\{\left[\sum_{m=1}^{M_{0}}\left|B_{m}\right|\right]^{2}\right\} .
$$

Further, we define factor $\beta$ to compare the output signal-tonoise power ratios in different $M$ as follows:

$$
\begin{aligned}
\beta & =\frac{\left.P R_{\text {out }}\right|_{M=M_{0}}}{\left.P R_{\text {out }}\right|_{M=1}} \\
& =\frac{E\left\{\left[\sum_{m=1}^{M_{0}}\left|A_{m}\right|\right]^{2}\right\}}{E\left\{\left|A_{1}\right|^{2}\right\}} / \frac{E\left\{\left[\sum_{m=1}^{M_{0}}\left|B_{m}\right|\right]^{2}\right\}}{E\left\{\left|B_{1}\right|^{2}\right\}} .
\end{aligned}
$$

It is noteworthy that, the factor $\beta$ is dependent on both the ratio of signal power and the ratio of noise power in different $M$. If $\beta>1$ in the case of $M_{0}>1$, a better noise-mitigation capability can be achieved for the proposed timing metric.

First, for the signal power ratio in (24), by exploiting the excellent properties of ZC sequence and the expression of $A_{m}$ in (14), we can obtain

$$
E\left\{\left[\sum_{m=1}^{M_{0}}\left|A_{m}\right|\right]^{2}\right\}=\left[\left|h_{l}\right|^{2} \sum_{m=1}^{M_{0}}(N-m)\right]^{2},
$$

and

$$
E\left\{\left|A_{1}\right|^{2}\right\}=\left[\left|h_{l}\right|^{2}(N-1)\right]^{2} .
$$

Consider the best trade-off between complexity and timing estimation performance, we assume the correlation length $M_{0} \ll N$ is enough for an acceptable performance. Therefore, the output signal power ratio is approximately $M_{0}^{2}$.

We now analyze the output noise power ratio in (24), which can be rewritten as

$$
\begin{aligned}
& \frac{E\left\{\left[\sum_{m=1}^{M_{0}}\left|B_{m}\right|\right]^{2}\right\}}{E\left\{\left|B_{1}\right|^{2}\right\}} \\
& =\frac{\sum_{m=1}^{M_{0}} E\left\{\left|B_{m}\right|^{2}\right\}}{E\left\{\left|B_{1}\right|^{2}\right\}}+\frac{\sum_{m=1}^{M_{0}} \sum_{n=1, n \neq m}^{M_{0}} E\left\{\left|B_{m}\right|\left|B_{n}\right|\right\}}{E\left\{\left|B_{1}\right|^{2}\right\}} .
\end{aligned}
$$

Since $B_{m}$ obeys the complex Gaussian distribution, $\left|B_{m}\right|$ is a Rayleigh random variable with mean

$$
E\left\{\left|B_{m}\right|\right\}=\sqrt{\frac{\pi}{4} \operatorname{Var}\left\{B_{m}\right\}}
$$

and variance

$$
\operatorname{Var}\left\{\left|B_{m}\right|\right\}=\frac{4-\pi}{4} \operatorname{Var}\left\{B_{m}\right\},
$$

respectively. In consideration of the relation between its mean and variance, we have $E\left\{\left|B_{m}\right|^{2}\right\}=\operatorname{Var}\left\{B_{m}\right\}$. Hence, 
the first term on the right hand side (RHS) of (27) can be calculated as

$$
\frac{\sum_{m=1}^{M_{0}} E\left\{\left|B_{m}\right|^{2}\right\}}{E\left\{\left|B_{1}\right|^{2}\right\}}=\frac{\sum_{m=1}^{M_{0}} \operatorname{Var}\left\{B_{m}\right\}}{\operatorname{Var}\left\{B_{1}\right\}} \approx M_{0} .
$$

Likewise, the second term on the RHS of (27) can be simplified as

$$
\begin{aligned}
& \frac{\sum_{m=1}^{M_{0}} \sum_{n=1, n \neq m}^{M_{0}} E\left\{\left|B_{m}\right|\left|B_{n}\right|\right\}}{E\left\{\left|B_{1}\right|^{2}\right\}} \\
& \approx \frac{M_{0}\left(M_{0}-1\right)\left[E\left\{\left|B_{1}\right|\right\}\right]^{2}}{E\left\{\left|B_{1}\right|^{2}\right\}} \\
& =\frac{\pi}{4}\left(M_{0}-1\right) M_{0} .
\end{aligned}
$$

As a consequence, the output noise power ratio can be given by substituting (30) and (31) to (27), i.e.

$$
E\left\{\left[\sum_{m=1}^{M_{0}}\left|B_{m}\right|\right]^{2}\right\} / E\left\{\left|B_{1}\right|^{2}\right\}=\frac{\pi}{4} M_{0}^{2}+\left(1-\frac{\pi}{4}\right) M_{0}
$$

Based on the above results, the factor $\beta$, used to reflect the enhancement of anti-noise capability for different correlation lengths, is finally determined as

$$
\beta=\frac{\left.P R_{\text {out }}\right|_{M=M_{0}}}{\left.P R_{\text {out }}\right|_{M=1}}=\frac{4}{\pi} \frac{1}{1+\frac{1}{M_{0}}\left(\frac{4}{\pi}-1\right)} .
$$

Note that, in (33), the value of $\beta$ is proportional to $M_{0}$, and always larger than one when $M_{0}>1$. It means that the output power ratio increases as the increment of correlation length. That is to say, with a flexibly adjusted correlation length, the proposed timing metric based on LDCC can effectively mitigate the impact of noise, and consequently will obtain a notably improved performance, even at low SNRs. In practical implement, on the premise of ensuring reliable timing estimation performance, the design of parameter $M$ is necessary to be carefully considered, so as to save computational overhead of the load-limited satellite receiver as much as possible.

2) Wrong timing position: According to the statistical analysis at the correct timing position, the proposed timing metric is verified to be able to effectively restrain the adverse impacts of large CFO and noise on preamble detection. However, in some scenarios with severe channel dispersion (e.g. the device is located at a densely built city with a small elevation angle), it may not work well because the strongest path corresponding to the maximum correlation peak may not be the first one, leading to a significant decrease in timing estimation accuracy. To resolve this problem, a pre-set detection threshold is required to track the time instants corresponding to the arriving paths, while to make correlation results of the other instants below it with an extremely high probability. Towards this end, we first conduct statistical analysis of the proposed timing metric at the wrong timing positions, and then design the first-path detection threshold in terms of the target false alarm probability.
At the wrong time instant $d_{w}$, the received signal in (2) will be only made up of noise term, thus we have

$$
r_{d_{w}}=w_{d_{w}}
$$

which always holds for an arbitrary $d_{w} \notin\left\{\tau+\tau_{l}, 0 \leq l \leq\right.$ $L-1\}$. Then, substituting (34) to (9) gives

$$
\begin{aligned}
T_{p}\left(d_{w}\right) & =\frac{P\left(d_{w}\right)}{R\left(d_{w}\right)}=\frac{\sum_{m=1}^{M} P_{m}\left(d_{w}\right)}{R\left(d_{w}\right)} \\
= & \frac{\sum_{m=1}^{M}\left|\sum_{n=1}^{N-m} S_{n}^{1}\left(S_{n+m}^{2}\right)^{*}\left(w_{n+d_{w}}^{1}\right)^{*} w_{n+d_{w}+m}^{2}\right|}{\sum_{m=1}^{M} \sum_{n=1}^{N-m}\left|\left(w_{n+d_{w}}^{1}\right)^{*} w_{n+d_{w}+m}^{2}\right|} .
\end{aligned}
$$

We first consider the normalization function in (35). Because $\left(w_{n+d_{c}}^{1}\right)^{*}$ and $w_{n+d_{c}+m}^{2}$ are i.i.d. complex Gaussian random variables, their product can be approximated as a complex Gaussian random variable with mean 0 and variance $\sigma_{w}^{2}$. Such that, $\left|\left(w_{n+d_{c}}^{1}\right)^{*} w_{n+d_{c}+m}^{2}\right|$ follows a Rayleigh distribution with mean $\sqrt{\pi / 4} \sigma_{w}$. Consequently, the mean of $R\left(d_{w}\right)$ is obtained as

$$
\begin{aligned}
E\left\{R\left(d_{w}\right)\right\} & =\sum_{m=1}^{M} \sum_{n=1}^{N-m} E\left\{\left|\left(w_{n+d_{w}}^{1}\right)^{*} w_{n+d_{w}+m}^{2}\right|\right\} \\
& =\frac{\sqrt{\pi}}{2} \sigma_{w}\left[M N-\frac{(M+1) M}{2}\right] .
\end{aligned}
$$

For the correlation function in (35), since the product of $S_{n}^{1}$ and $\left(S_{n+m}^{2}\right)^{*}$ is an exponential factor, each term in $P_{m}\left(d_{w}\right)$ is virtually the phase rotation of $\left(w_{n+d_{w}}^{1}\right)^{*} w_{n+d_{w}+m}^{2}$, which means the probability distribution of $P_{m}\left(d_{w}\right)$ only depends on the pure noise component. As aforementioned, $\left(w_{n+d_{c}}^{1}\right)^{*} w_{n+d_{c}+m}^{2}$ obeys a complex Gaussian distribution, and consequently the sum of $(N-m)$ i.i.d. complex Gaussian random variables, $P_{m}\left(d_{w}\right)$, still follows a complex Gaussian distribution and its variance is

$$
\operatorname{Var}\left\{P_{m}\left(d_{w}\right)\right\}=(N-m) \sigma_{w}^{2} .
$$

As a result, $\left|P_{m}\left(d_{w}\right)\right|$ is a Rayleigh random variable with mean

$$
E\left\{\left|P_{m}\left(d_{w}\right)\right|\right\}=\sqrt{\frac{\pi}{4}(N-m)} \sigma_{w},
$$

and variance

$$
\operatorname{Var}\left\{\left|P_{m}\left(d_{w}\right)\right|\right\}=\frac{4-\pi}{4}(N-m) \sigma_{w}^{2} .
$$

Based on the above derivation, we further determine the detection threshold. The false alarm probability, $P_{F A}$, is denoted as the probability that the value of timing metric in the absence of two preamble sequences exceeds a pre-defined detection threshold, $\gamma$, and can be expressed as

$$
P_{F A}=P_{b}\left(T_{p}\left(d_{w}\right)>\gamma\right)
$$

where $P_{b}(\cdot)$ represents the probability of a random event. Similar to [32], we replace $R\left(d_{w}\right)$ with its mean, and (40) is rewritten as

$$
\begin{aligned}
P_{F A} & \approx P_{b}\left(P\left(d_{w}\right) / E\left\{R\left(d_{w}\right)\right\}>\gamma\right) \\
& =P_{b}\left(\sum_{m=1}^{M}\left|P_{m}\left(d_{w}\right)\right|>\gamma E\left\{R\left(d_{w}\right)\right\}\right) .
\end{aligned}
$$


It is notable from (41) that $P\left(d_{w}\right)$ is the sum of $M$ Rayleigh random variables, however, its cumulative distribution function (CDF) does not have a closed-form solution [33], [34]. Such that, the accurate expression of $P_{F A}$ will be a laborious task, which further makes the determination of a reasonable detection threshold much more difficult. Fortunately, we can utilize the summation property of probability inequations to resolve this problem, i.e.

$$
\begin{aligned}
& P_{b}\left(\sum_{m=1}^{M}\left|P_{m}\left(d_{w}\right)\right|>\gamma E\left\{R\left(d_{w}\right)\right\}\right) \\
& \leq \sum_{m=1}^{M} P_{b}\left(\left|P_{m}\left(d_{w}\right)\right|>\frac{\gamma E\left\{R\left(d_{w}\right)\right\}}{M}\right) .
\end{aligned}
$$

By substituting (42) to (41) while considering the probability density function (PDF) of Rayleigh random variable, we have

$$
\begin{aligned}
P_{F A} & \leq \sum_{m=1}^{M} \exp \left[-\left(\frac{E\left\{R\left(d_{w}\right)\right\} \gamma}{M}\right)^{2} / \operatorname{Var}\left\{P_{m}\left(d_{w}\right)\right\}\right] \\
& \leq M \exp \left[-\left(\frac{E\left\{R\left(d_{w}\right)\right\} \gamma}{M}\right)^{2} / \operatorname{Var}\left\{P_{1}\left(d_{w}\right)\right\}\right] .
\end{aligned}
$$

Further, by exploting (36) and (37), the above inequation can be further derived as

$$
P_{F A} \leq P_{F A_{\max }} \triangleq M \exp \left[-\frac{\pi(2 N-M-1)^{2}}{16(N-1)} \gamma^{2}\right],
$$

where $P_{F A_{\max }}$ is defined as the tolerated maximum value of false alarm probability. Therefore, as long as $P_{F A_{\max }}$ is given (for instance, $P_{F A_{\max }}=0.1 \%$ in 5G RA), the detection threshold $\gamma$ can be finally achieved as

$$
\gamma=\sqrt{-\frac{16(N-1)}{\pi(2 N-M-1)^{2}} \ln \left(\frac{P_{F A_{\max }}}{M}\right)} .
$$

It is clear that the determination of threshold is only dependent on the correlation length $M$ without the priori knowledge of statistical properties of noise, which is usually calculated by a maximum likelihood estimator or approximated by the samples of power delay profile in the traditional methods. This remarkable improvement is mainly attributed to the ingenious design of timing metric that can eliminate the influence of noise variance on determining $\gamma$. Furthermore, because the setting of threshold does not need noise variance estimation, the computational efficiency is also enhanced during the procedure of RA preamble detection.

\section{Proposed Timing Estimation Method}

Based on the statistical analysis of the proposed timing metric at different timing positions, the corresponding timing estimation method for satellite multi-path fading channels can be concluded as follows:

$$
\begin{gathered}
\widehat{d}=\underset{d}{\arg \max }\left\{T_{p}(d)\right\} \\
\widehat{d}_{\text {opt }}=\underset{d_{\text {path }}}{\arg \text { first }}\left\{T_{p}\left(d_{\text {path }}\right)>\gamma\right\}, d_{\text {path }} \in\left\{\widehat{d}-\tau_{\text {max }}, \widehat{d}\right\}
\end{gathered}
$$

TABLE I

Parameter Configurations in LEO SATEllite Scenario.

\begin{tabular}{c|c}
\hline Parameters & Configurations \\
\hline Band & S-band \\
Carrier frequency & $2 \mathrm{GHz}$ \\
Satellite altitude & $1200 \mathrm{~km}$ \\
Beam set & Sat-2 \\
Elevation angle & $45 \mathrm{degree}$ \\
Max differential delay & $1.7 \mathrm{~ms}$ \\
Max Doppler shift & $40 \mathrm{KHz}$ \\
RACH bandwidth & $1.08 \mathrm{MHz}$ \\
Sub-carrier interval & $1.25 \mathrm{KHz}$ \\
Correlation length, $M$ & {$[1,2,4,8,16]$} \\
Channel model & AWGN / FSF \\
\hline \\
$\gamma=\sqrt{-\frac{16(N-1)}{\pi(2 N-M-1)^{2}} \ln \left(\frac{P_{F A_{\max }}}{M}\right)}$
\end{tabular}

In (46), $\widehat{d}$ denotes the timing position corresponding to the strongest arrival path, which can be obtained by capturing the maximum value of the timing metric. If $\widehat{d}$ is determined, the ideal timing position $\widehat{d}$, corresponding to the first arrival path, is ensured to exist in the range of the channel delay spread $\left\{0, \tau_{\max }\right\}$. Hence, by searching forward $d_{\text {path }}$ corresponding to the first value of the timing metric greater than the predefined threshold, $\widehat{d}_{o p t}$ can be finally achieved as shown in (47). Here, to achieve a reliable timing estimation, the threshold $\gamma$ in (48) could be correspondingly chosen according to the correlation length and the desired probability of false alarm.

\section{Performance Evaluation and Discussions}

To assess the timing performance of our method by computer simulation, we consider a 5G-enabled LEO satellite RA scenario reported in the latest 3GPP TR 38.821 [22], and Table I lists the detailed configurations of system parameters. In this scenario, the max differential delay is $1.7 \mathrm{~ms}$, and thus the $\mathrm{CP}$ duration $T_{C P}$ must be greater than or equal to twice of this value to avoid ISI, such that we set $T_{C P}$ to be $3.4 \mathrm{~ms}$. To satisfy the coverage performance within the beam, the duration of E-SCLS, $T_{L S}$, is calculated based on the link budget [17] as $6.4 \mathrm{~ms}$. In consideration of system compatibility, the subcarrier interval is set to be the value adopted in LTE/NR PRACH, i.e. $1.25 \mathrm{KHz}$, and consequently the duration $T_{S E Q}$ of sub-sequence of E-SCLS is $0.8 \mathrm{~ms}$. That is to say, the proposed E-SCLS consists of $K=8$ short ZC sequences with length of $N=839$. Further, for preamble sequence design, the root index $u$ can be flexibly chosen from the set $\{1,839\}$, and the cyclic shift offset $N_{C S}$ is configured to be 101. Here, both AWGN and frequency selective fading (FSF) channels are utilized for performance evaluation of the proposed method.

\section{A. Performance Validation of The proposed Method}

First, we evaluate the performance of the proposed method in AWGN channel, so as to validate the effectiveness of theoretical analysis in Section III. In Fig. 6, we show the simulated and analytical results of the output power ratio of 


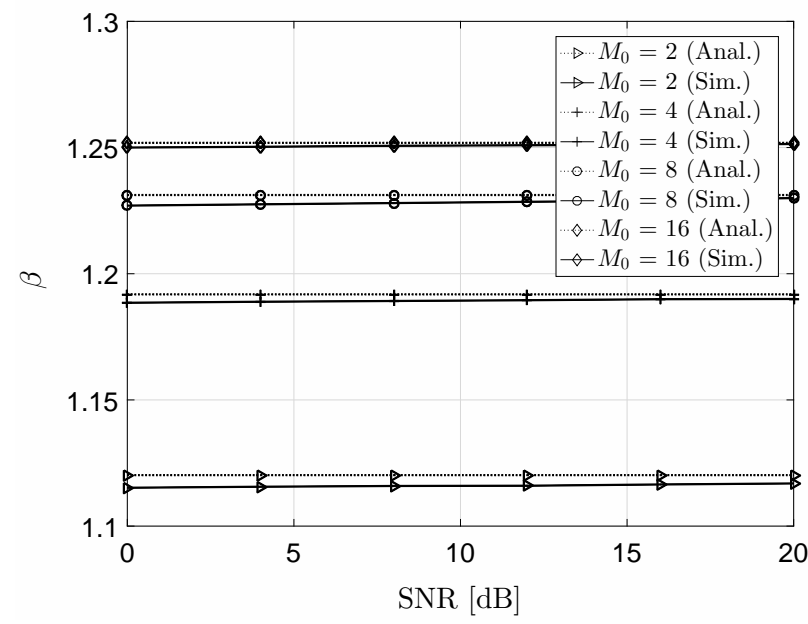

Fig. 6. Output power ratio of signal to noise versus SNR for different number of correlation rows.

signal to noise, $\beta$, for different number of correlation rows, $M_{0}$. We notice that the numerical results nearly coincide with the analytical results for different $M_{0}$, which demonstrates the validity of statistical derivation at the correct timing position, and the inappreciable difference between them for the same $M_{0}$ is mainly due to the approximation of the noise statistics. Furthermore, it can be also seen from Fig. 6 that, $\beta$ is always larger than 1 and merely increases with the number of correlation rows, which fully shows that the noise-mitigation capability of the proposed method can be further enhanced with the increment of the number of correlation rows.

We adopt the mean square error (MSE) as the evaluative criteria of timing estimation, and investigate the timing performance of our method versus SNR with different correlation lengths used in LDCC. As shown in Fig. 7, the timing accuracy gradually enhances with the increment of correlation length, since the proposed timing metric with larger $M$ is able to obtain a better anti-noise capability. Moreover, it is evident that the amplitude gain between the MSE curves with different $M$ becomes higher as SNR increases, for instance, the gain between two MSE curves with $M=16$ and $M=1$ can reach to be greater than $10^{3}$ at $\mathrm{SNR}=-10 \mathrm{~dB}$. That is to say, our method can achieve a significantly improved the timing estimation performance by increasing the correlation length. Here, it is worth noting that computational complexity is also one of the key issues in the practical implement of the satellite station. If the complexity is the main concern, we could choose relatively less correlation rows in LDCC, e.g. $M=1$ or $M=2$; otherwise, we configure $M=4$ or $M=8$ for achieving a better timing estimation performance, on condition that the system can afford such complexity.

Further, we continue to study the impact of CFO on the proposed timing estimation. Fig. 8 depicts the timing MSE curves in various normalized $\mathrm{CFO}, \varepsilon$, wherein the proposed method and DCC as well as the existing methods in [19], [20], and [21] are illustrated at SNR $=-11 \mathrm{~dB}$. Although DCC is immune to CFO, its performance is seriously affected by noise, and thus it has a considerable timing MSE in low SNR. Thanks to having a larger sub-carrier spacing, our previous method

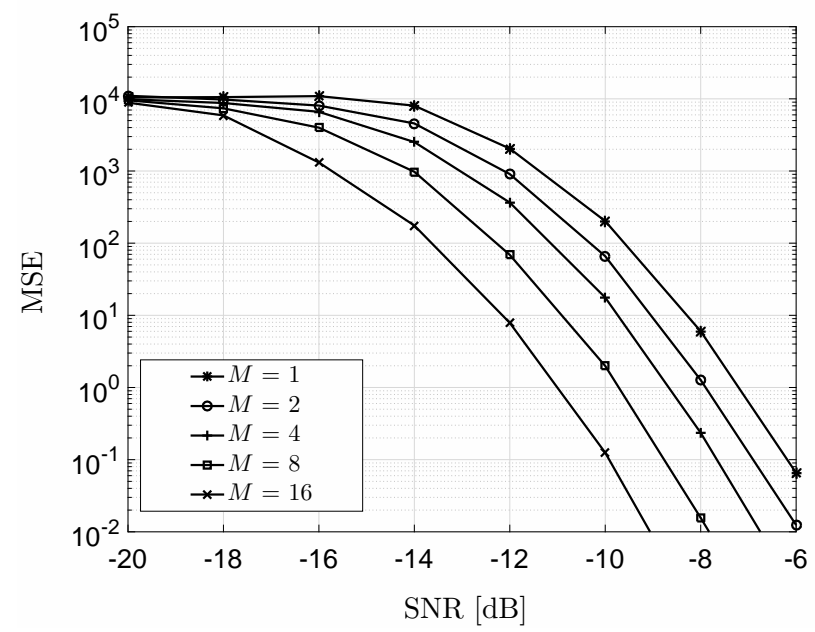

Fig. 7. Timing estimation performance of the proposed method with different correlation lengths in LDCC.

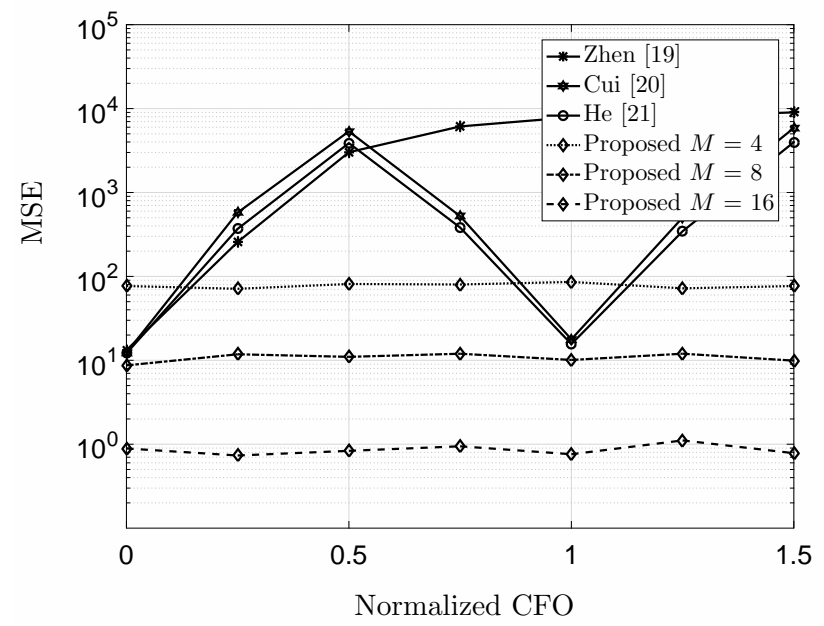

Fig. 8. Timing estimation performance of the proposed method in different CFOs.

[19] can effectively work in case that CFO is lower than half of the sub-carrier spacing, however, its estimation accuracy becomes worse as $\varepsilon$ increases. For both the methods in [20], and [21], a relatively lower timing MSE can be achieved only in the presence of integral $\varepsilon$, but their performance experience severe degradation if the fractional $\varepsilon$ exists, and will get worst when CFOs are exactly the multiples of half of the subcarrier spacing. By contrast, the proposed method obtains a stable MSE performance versus different CFOs, because the influence of CFO can be effectively eliminated by modulus operation of the timing metric in (7). Besides, its performance could be also improved with the increment of $M$, and notably outperforms the other methods in the case of $M=16$.

\section{B. Performance Comparisons With The Existing Methods}

In order to reveal the feasibility of the proposed method in different satellite communication environments, we make performance comparisons with the related works under two FSF channels in [35]. Here, a 7-paths Rician channel is used to describe the satellite LOS scenario (e.g. the access device is located at a country/suburban or has a large elevation angle), in 


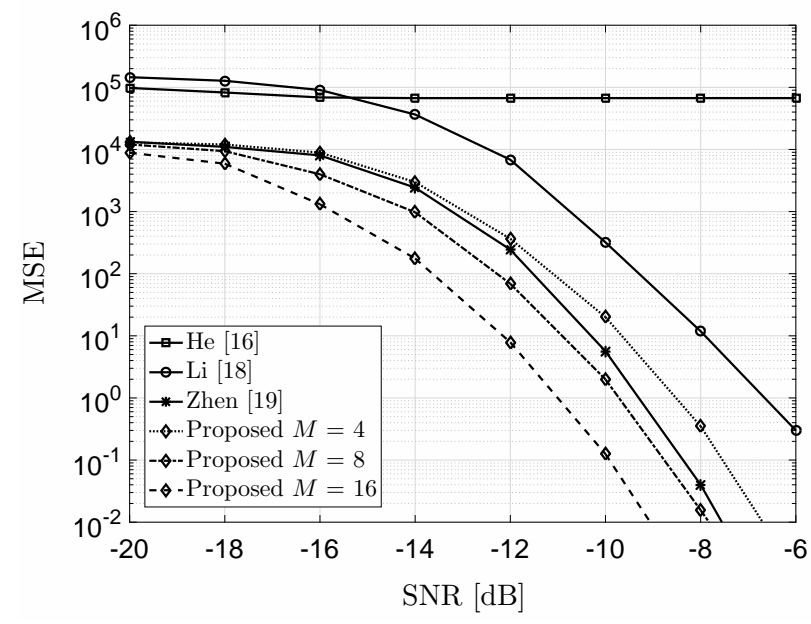

Fig. 9. Timing MSE performance of different RA methods in a 7-paths Rician channel.

which relative path delays and path gains are set as $\{0,195.3$, $260.4,846.3,1171.9,1953.1,2734.3\}[\mathrm{ns}]$ and $\{-91.9,106.3$, $-110.1,-112.5,-110.2,-112.5,-112.5\}[\mathrm{dBm}]$, respectively. The magnitude of the first path obeys Rician distribution with factor 10, and those of the others are Rayleigh distributed. On the other hand, we adopt a 4-paths Rayleigh channel to emulate the satellite NLOS scenario (e.g. the access device is located at a densely built city or has a small elevation angle) with relative path delays $\{0,195.3,260.4,390.6\}[\mathrm{ns}]$ and path gains $\{-108.5,-110.9,-106.6,-109.3\}[\mathrm{dBm}]$. In the following, we perform performance comparisons for different RA methods from the aspects of timing MSE, the success probability of first access, and the mean normalized access energy, respectively. Notice that the preamble designs of the referenced methods are based on the system parameters listed in Table I.

Fig. 9 exhibits the timing MSE performance curves of different RA methods in a 7-paths Rician channel. Here, the residual $\mathrm{CFO}$ is $250 \mathrm{~Hz}$ after frequency compensation, and the target false alarm probability is set to be the same value specified in 5G RA, i.e. $10^{-3}$. It can be seen from Fig. 9 that, since the timing estimation performance in [16] with small sub-carrier spacing is greatly affected by $\mathrm{CFO}$, this method suffers from a considerable timing error at different SNRs. By reusing the terrestrial 5G RA preamble format in [18] and [19], both methods have a relatively larger sub-carrier interval compared to that in [16], and thus could be effectively used for tracking the first and strongest arrival path in satellite LOS scenario. It is notable that, the timing estimation performance of the proposed method can be continuously improved by increasing the correlation length. To clarify, at an appropriately correlation length, that is when $M$ is set as 8 or 16 , the proposed method can achieve a significantly lower timing MSE compared to the existing methods.

Further, we compare the timing MSE performance of the aforementioned methods under a 4-paths Rayleigh channel in Fig. 10. Here, the CFO and the target false alarm probability are set as 0 and $10^{-3}$, respectively. Note that all the methods have high timing errors at extremely low SNRs;

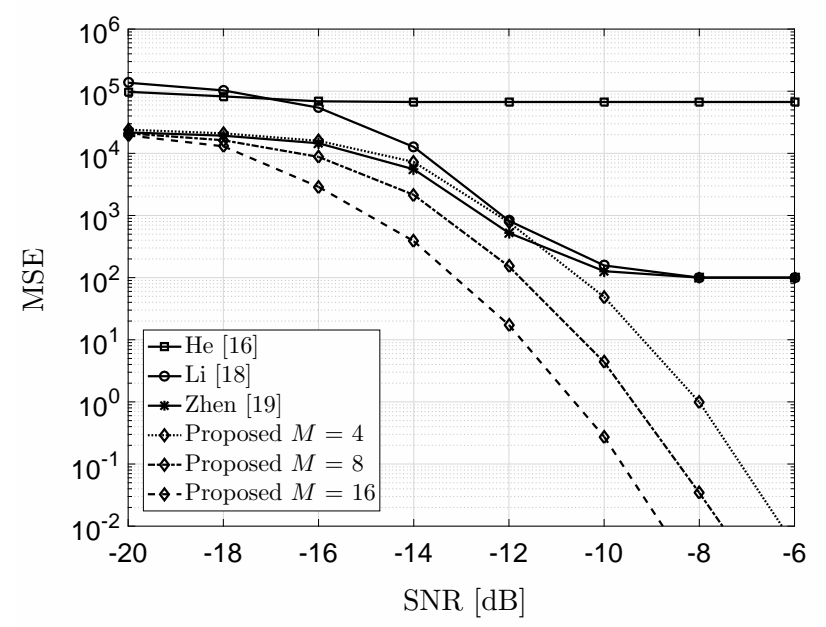

Fig. 10. Timing MSE performance of different RA methods in a 4-paths Rayleigh channel.

however, with the increment of SNR, the influence of multipath effect dominates, which may greatly affect their timing estimation performance. To be specific, there is no further performance improvement for the methods in [18] and [19] when SNR $>-10 \mathrm{~dB}$. This is because both methods can only indicate the strongest LOS path, which is not the first arriving path in this satellite NLOS scenario. On the contrary, due to the reasonable design of detection threshold, the proposed method can effectively track the first path, and consequently obtain a much better timing accuracy in contrast with the conventional methods. Again, its performance is also improved as the correlation length increases.

As we know, the success probability of channel access is one of vital evaluation criteria of access efficiency in satellite RA, which can be utilized to indicate energy efficiency [36], [37] and access delay. To demonstrate the performance benefit of channel access success probability, we compare the proposed method with our earlier method given in [19], wherein CFO is set to 0 for fair comparison. As shown in Fig. 11, it is observed that our method in [19] could achieve an acceptable success probabilities in both AWGN channel and 7-paths Rician channel. However, in the 4-paths Rayleigh channel, its performance is extremely degraded due to the existence of stronger scattering paths. By contrast, attributable to the efficient resistance of multi-path effect, the proposed method has an obviously higher success probabilities in different channel conditions.

We shall now evaluate the energy efficiency of our proposed method. The energy efficiency of our proposed method is directly due to the high access probability which keeps the number of attempts for a successful channel access low. We show the mean normalized access energy for each method, which is the energy consumption for the channel access normalized to the energy consumption of a single access. The average energy consumed for a successful channel access depends on the access probability. Since each channel access attempt is independent, the channel access attempt can be described by a Geometric process, and mean number of attempts for a success access can be determined. Given that each 


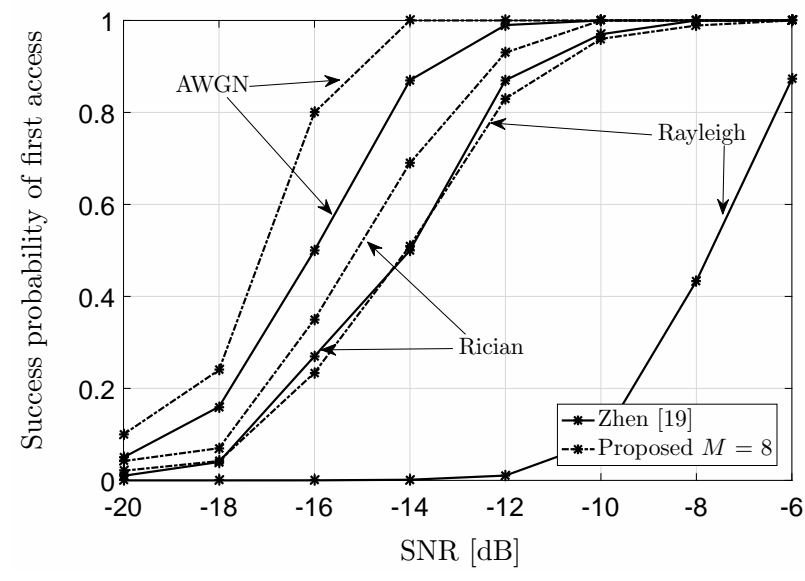

Fig. 11. Performance comparisons of success probability of first access in different channel conditions.

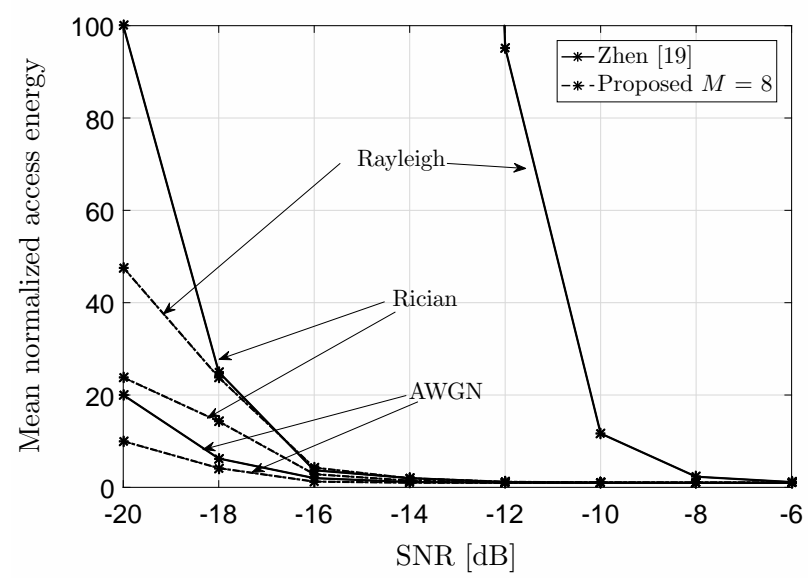

Fig. 12. Performance comparisons of mean normalized access energy in different channel conditions.

channel access consumes the same energy, the mean number of a successful attempt can be used directly to describe the mean normalized energy consumption [38]. To illustrate the energy efficiency of the proposed method in various satellite environments, we again compare the proposed method with our previously presented method [19] for different channel conditions in Fig. 12. Likewise, CFO is set to 0 for fair comparison. We immediately see adequately low normalized energy consumption for the proposed scheme for the channel access. The results further indicate that the proposed method can be applied to various satellite environments, regardless of severe channel dispersion.

\section{Discussions and Suggestions}

Based on the above simulation results, we have shown the robustness of the proposed method to severe channel distortions. In this section, we further discuss and summarize the characteristics of our methods in [19] and in this paper, and give some suggestions on how to choose a more appropriate method according to the real satellite communication scenario.

As shown in Table II, by adopting SCLS as the preamble sequence, our previous method only uses the least root ZC sequences for generating all the available sequences, and thus
TABLE II

PERFORMANCE COMPARISONS

\begin{tabular}{c|c|c}
\hline Approach & {$[19]$} & Proposed method \\
\hline Preamble sequence & SCLS & E-SCLS \\
Number of root sequences & Few & Many \\
Computational complexity & Low & High \\
Interference-suppression capability & Strong & Weak \\
CFO-resistance capability & Weak & Strong \\
Noise-mitigation capability & Weak & Strong \\
Anti-multipath fading capability & Weak & Strong \\
\hline
\end{tabular}

the correlation peaks of access users exploiting the same root $\mathrm{ZC}$ sequence can be obtained by performing one-shot PDP calculation in the detection window. Hence, this method can have a extremely low detection complexity and a remarkable non-orthogonal interference (caused by the correlation between different root $\mathrm{ZC}$ sequences)-suppression capability. On the other hand, for the proposed method that adopts ESCLS, each available RA preamble has its own root ZC sequence, and consequently a larger number of root sequences is required to generate all the available sequences. Such that, a relatively higher detection complexity is achieved compared with that in [19], since each used root sequence needs one time correlation detection. Meanwhile, in the case of massive users access, interference-suppression capability of the proposed method gradually weakens as the number of users increases. Nevertheless, because the timing metric based on LDCC is not only robust to CFO, but also has a the flexibly adjusted correlation length, while considering the elaborately design of first-path detection threshold that independent of noise statistics, the proposed method is distinctly superior to [19] in terms of CFO-resistance capability, noise-mitigation capability, and anti-multipath fading capability.

In summation, according to the actual communication scenario parameters (such as user location, elevation angle, and etc.) and the available computing resources, the satellite receiver can adaptively choose an appropriate method based on the own unique advantages of two methods. For instance, if the access device is located at a country/suburban or has a large elevation angle, LOS path exists and Doppler shift is relatively lower, the previous method could be chosen for RA preamble design and detection; in case the access device is located at a densely built city or has a small elevation angle, NLOS scenario with large Doppler shift may occur, it is more reliable to choose the proposed method in this paper.

\section{CONCLUSION}

In this paper, we focused on the robust design and detection of RA preamble for high-efficiency LEO satellite-assisted 6G IoT. First, we proposed an enhanced preamble sequence, referring to as E-SCLS, by concatenating the conjugated and circularly shifted replicas of a single root $\mathrm{ZC}$ sequence, so as to perform one-step TA estimation to avoid additional signaling overhead and detection procedure. Then, we presented a novel timing metric based on LDCC that can achieve the immunity to CFO and mitigation of noise, and revealed its superiority according to the statistical analysis at the correct timing index. We further derived the first-path detection threshold according 
to the target false alarm probability and finally showed the proposed timing estimation method for satellite multi-path fading channels.

Numerical results in different LEO scenarios validated the effectiveness of statistical analysis, and demonstrated that the proposed method with the robustness to severe channel distortion can significantly improve both the access performance and efficiency as compared to the state-of-the-art methods, in terms of timing MSE, success probability of first access, and mean normalized access energy. Moreover, we have also summarized the characteristics of our methods in [19] and in this paper, and given suggestions with respect to choosing a more appropriate method based on the actual communication scenario parameters. Note that, since each available preamble employs an individual root $\mathrm{ZC}$ sequence in the proposed method, the utilization of extra root sequences will introduce a high detection complexity as well as non-orthogonal interference in the case of massive access. In the future work, it is of great interest to further design the related successive interference cancellation (SIC) method with an acceptable complexity.

\section{REFERENCES}

[1] B. Aazhang, P. Ahokangas, M. S. Alouini, and P. Zhu, "Key drivers and research challenges for $6 \mathrm{G}$ ubiquitous intelligence (white paper)," $6 \mathrm{G}$ Flagship, Univ. Oulu, Oulu, Finland, Sep. 2019.

[2] W. Saad, M. Bennis, and M. Chen, "A vision of $6 \mathrm{G}$ wireless systems: Applications, trends, technologies, and open research problems," IEEE Netw., vol. 34, no. 3, pp. 134-142, May 2020.

[3] B. Zong, C. Fan, X. Wang, X. Duan, B. Wang, and J. Wang, "6G technologies: Key drivers, core requirements, system architectures, and enabling technologies," IEEE Veh. Technol. Mag., vol. 14, no. 3, pp. 18-27, Sep. 2019.

[4] P. Yang, Y. Xiao, M. Xiao, and S. Li, "6G wireless communications: Vision and potential techniques," IEEE Netw., vol. 33, no. 4, pp. 70-75, Jul. 2019.

[5] M. De Sanctis, E. Cianca, G. Araniti, I. Bisio, and R. Prasad, "Satellite communications supporting Internet of remote things," IEEE Internet Things J., vol. 3, no. 1, pp. 113-123, Feb. 2016.

[6] Study on RAN Improvements for Machine-type Communications, 3GPP TR 37.868, 2011.

[7] E. Park, J. Bae, Y. Choi, and Y. Han, "Energy-efficient random access for LTE-based stationary IoT networks," IEEE Commun. Lett., vol. 23, no. 2, pp. 346-349, Feb. 2019.

[8] E. Park, J. Bae, Y. Choi, and Y. Han, "A novel energy efficient random access response for IoT networks," in Proc. IEEE Veh. Technol. Conf. (VTC), Chicago, USA, Aug. 2018.

[9] S. Shailendra, A. R. K, B. Panigrahi, H. K. Rath, and A. Simha, "Power efficient RACH mechanism for dense IoT deployment," in Proc. IEEE Int. Conf. on Commun. (ICC), Paris, France, May 2017.

[10] H. S. Jang, B. C. Jung, and D. K. Sung, "Dynamic access control with resource limitation for group paging-based cellular IoT systems," IEEE Internet Things J., vol. 5, no. 6, pp. 5065-5075, Dec. 2018.

[11] Z. Zhou, Y. Guo, Y. He, X. Zhao, and W. M. Bazzi, "Access control and resource allocation for M2M communications in industrial automation," IEEE Trans. Ind. Informat., vol. 15, no. 5, pp. 3093-3103, May 2019.

[12] J. Misic and V. B. Misic,"Efficiency of power ramping during random access in LTE," IEEE Trans. Veh. Technol., vol. 67, no. 2, pp. 1698-1712, Feb. 2018.

[13] F. H. S. Pereira, C. A. Astudillo, T. P. C. de Andrade, and N. L. S. da Fonseca, "PRACH power control mechanism for improving randomaccess energy efficiency in Long Term Evolution," in Proc. IEEE LatinAmerican Conf. on Commun. (LATINCOM), Guadalajara, Mexico, Nov. 2018.

[14] S. L., F. Qin, Z. Gao, Y. Zhang, and Y. He, "LTE-satellite: Chinese proposal for satellite component of IMT-Advanced system," China Commun., vol. 10, no. 10, pp. 47-64, Oct 2013.

[15] A. Guidotti, A. Vanelli-Coralli, M. Caus, J. Bas, G. Colavolpe, T. Foggi, S. Cioni, A. Modenini, and D. Tarchi, "Satellite-enabled LTE systems in LEO constellations," in Proc. IEEE Int. Conf. on Commun. (ICC), Paris, France, May 2017.
[16] Y. He, G. Cui, P. Li, R. Chang, and W. Wang, "Random access preamble design based on time pre-compensation for LTE-satellite system," $J$. China Univ. Posts Telecommun., vol. 22, no. 3, pp. 64-73, Jun. 2015.

[17] S. Sesia, M. Baker, and I. Toufik, "LTE-The UMTS long term evolution: from theory to practice," Hoboken, NJ, USA: Wiley, 2011.

[18] C. Li, H. Ba, H. Duan, Y. Gao, and G. Wu, "A two-step time delay difference estimation method for initial random access in satellite lte system," in Proc. Int. Conf. on Adv. Commun. Technol., Pyeongchang, South Korea, Feb. 2014.

[19] L. Zhen, H. Qin, B. Song, R. Ding, X. Du, and M. Guizani, "Random access preamble design and detection for mobile satellite communication systems," IEEE J. Sel. Areas Commun., vol. 36, no. 2, pp. 280-291, Feb. 2018.

[20] G. Cui, Y. He, P. Li, and W. Wang, "Enhanced timing advanced estimation with symmetric zadoff-chu sequences for satellite systems," IEEE Commun. Lett., vol. 19, no. 5, pp. 747-750, May 2015.

[21] Y. He, G. Cui, P. Li, R. Chang, and W. Wang, "Timing advanced estimation algorithm of low complexity based on DFT spectrum analysis for satellite system," China Commun., vol. 12, no. 4, pp. 140-150, Apr. 2015.

[22] Solutions for NR to support non-terrestrial networks (NTN), 3GPP TR 38.821, Dec. 2019.

[23] N. H. Mahmood, H. Alves, O. A. Lopez, M. Shehab, D. P. M. Osorio, and M. L.-Aho, "Six key features of machine type communication in 6G," in Proc. 6G Wireless Summit (6G SUMMIT), Levi, Finland, May 2020.

[24] M. A. Basset, R. Mohamed, M. Elhoseny, A. K. Bashir, A. Jolfaei, and N. Kumar, "Energy-aware marine predators algorithm for task scheduling in IoT-based fog computing applications," IEEE Trans. Ind. Informat., 2020.

[25] H. Liao, Z. Zhou, X. Zhao, S. Mumtaz, A. Jolfaei, S. H. Ahmed, and A. $\mathrm{K}$. Bashir, "Learning-based context-aware resource allocation for edge computing-empowered industrial IoT,' IEEE Internet Things J., vol. 7, no. 5 , pp. 4260-4277, May 2020 .

[26] L. Zhen, H. Qin, Q. Zhang, Z. Chu, G. Lu, J. Jiang, and M. Guizani, "Optimal preamble design in spatial group-based random access for satellite-M2M communications," IEEE Wireless Commun. Lett., vol. 8, no. 3, pp. 953-956, Jun. 2019.

[27] M. Hua, M. Wang, W. Yang, X. You, F. Shu, J. Wang, W. Sheng, and Q. Chen, "Analysis of the frequency offset effect on random access signals," IEEE Trans. on Commun., vol. 61, no. 11, pp. 4728-4740, Nov. 2013.

[28] M. Hua, M. Wang, K. W. Yang, and K. J. Zou, "Analysis of the frequency offset effect on zadoff-chu sequence timing performance," IEEE Trans. on Commun., vol. 62, no. 11, pp. 4024-4039, Nov. 2014.

[29] H. Abdzadeh-Ziabari and M. G. Shayesteh, "Robust timing and frequency synchronization for OFDM systems," IEEE Trans. Veh. Technol., vol. 60, no. 8, pp. 3646-3656, Jul. 2011.

[30] Y. Liu, H. Yu, F. Ji, F. Chen, and W. Pan, "Robust timing estimation method for OFDM systems with reduced complexity," IEEE Commun. Lett., vol. 18, no. 11, pp. 1959-1962, Nov. 2014.

[31] P. Bromiley, "Products and convolutions of Gaussian probability density functions," Tina-Vis. Memo, vol. 3, pp. 1-13, 2003.

[32] H. Abdzadeh-Ziabari and M. G. Shayesteh, "Sufficient statistics, classification, and a novel approach for frame detection in OFDM systems," IEEE Trans. Veh. Technol., vol. 62, no. 6, pp. 2481-2495, Jul. 2013.

[33] J. Hu and N. C. Beaulieu, "Accurate simple closed-form approximations to Rayleigh sum distributions and densities," IEEE Commun. Lett., vol. 9, no. 2, pp. 109-111, Feb. 2005.

[34] G. K. Karagiannidis, T. A. Tsiftsis, and N. C. Sagias, "A closedform upper-bound for the distribution of the weighted sum of Rayleigh variates," IEEE Commun. Lett., vol. 9, no. 7, pp. 589-591, Jul. 2005.

[35] Evaluation of the OFDM as a satellite radio interface, ETSI TR 102 443, Aug. 2008.

[36] W. Hao, Z. Chu, F. Zhou, S. Yang, G. Sun and K. Wong, "Green communication for NOMA-based CRAN," IEEE Internet Things J., vol. 6, no. 1, pp. 666-678, Feb. 2019.

[37] W. Hao et al., "Energy-efficient hybrid precoding design for integrated multicast-unicast millimeter wave communications with SWIPT," IEEE Trans. Veh. Technol., vol. 68, no. 11, pp. 10956-10968, Nov. 2019.

[38] F. Chiti, R. Fantacci, and L. Pierucci, "Energy efficient communications for reliable IoT multicast 5G/Satellite services," Future Internet, vol. 11, no. 8, Aug. 2019. 


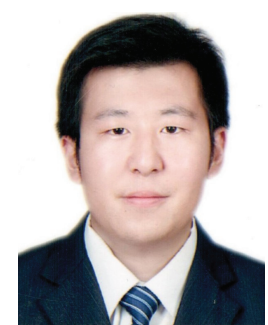

Li Zhen (Member, IEEE) received the M.S. degree in circuit and system from the Xi'an University of Posts and Telecommunications, Xi'an, China, in 2012, and the Ph.D. degree in communication and information systems from Xidian University, Xi' an, China, in 2018. He is currently a Lecturer with the School of Communication and Information Engineering, Xi' an University of Posts and Telecommunications, Xi'an, China. His research interests include $6 \mathrm{G}$ wireless communications, satellite mobile communications, machine-type communication$\mathrm{s}$, weak signal detection and processing, and random multiple access.

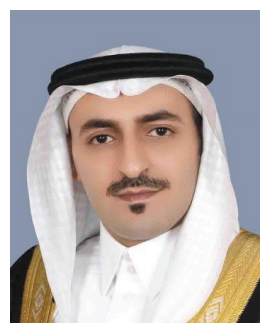

Yasser D. Al-Otaibi is currently an Assistant Professor in the Department of Information Systems at the Faculty of Computing and Information Technology in Rabigh, King Abdulaziz University, Jeddah, Saudi Arabia. He received a $\mathrm{PhD}$ degree in Information Systems from Griffith University, Australia in 2018. His current research interests include IT adoption and acceptance, wireless sensor networks, and IoT.

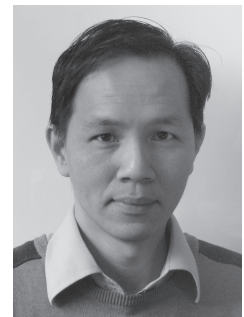

Chuan Heng Foh (Senior Member, IEEE) received the M.Sc. degree from Monash University, Australia, in 1999, and the Ph.D. degree from the University of Melbourne, Australia, in 2002. After his Ph.D. degree, he spent six months as a Lecturer at Monash University. From 2002 to 2012, he was an Assistant Professor with Nanyang Technological University, Singapore. He is currently a Senior Lecturer with the University of Surrey. He has authored or coauthored over 100 refereed articles in international journals and conferences. His research interests include protocol design and performance analysis of various computer networks, including wireless local area and mesh networks, mobile ad hoc and sensor networks, the Internet of Things, 5G networks, and data center networks. From 2015 to 2017, he has served as the Vice-Chair (Europe/Africa) of the IEEE Technical Committee on Green Communications and Computing (TCGCC). He is also an Associate Editor of IEEE Access and the IEEE Wireless Communications. Setropolitan Government, South Korea. He has worked on several research and industrial projects of South Korean, Japanese and European agencies and Government Ministries.

He received his Ph.D. in computer science and engineering from Korea University South Korea. He has authored over 140 research articles and is supervising/co-supervising several graduate (MS and $\mathrm{PhD}$ ) students. His research interests include internet of things, wireless networks, distributed systems, network/cyber security, cloud/network function virtualization, machine learning, etc. He is serving as the Editor-in-chief of the IEEE FUTURE DIRECTIONS NEWSLETTER. He is also serving as area editor of KSII Transactions on Internet and Information Systems; associate editor of IEEE Access, IET Quantum Computing. He is leading many conferences as a chair (program, publicity, and track) and had organized workshops in flagship conferences like IEEE Infocom, IEEE Globecom, IEEE Mobicom, etc.

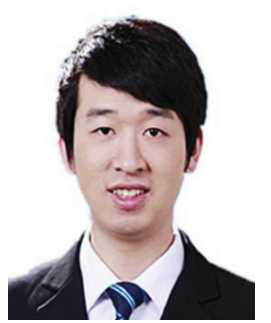

Keping Yu (Member, IEEE) received the M.E. and Ph.D. degrees from the Graduate School of Global Information and Telecommunication Studies, Waseda University, Tokyo, Japan, in 2012, and 2016, respectively.

He was a Research Associate and a Junior Researcher with the Global Information and Telecommunication Institute, Waseda University, from 2015 to 2019 and from 2019 to 2020 , respectively, where he is currently a Researcher. He has hosted and participated in more than ten projects, is involved in many standardization activities organized by ITU-T and ICNRG of IRTF and has contributed to ITU-T Standards Y.3071 and Supplement 35. His research interests include smart grids, information-centric networking, the Internet of Things, blockchain, and information security. He was the Chair of the IEEE/CIC ICCC 2nd EBTSRA workshop, the General Co-Chair and the Publicity Co-Chair of the IEEE VTC2020-Spring EBTSRA workshop, the TPC Co-Chair of the SCML2020, the Local Chair of the MONAMI 2020 , the Session Co-Chair of the CcS2020, and the Session Chair of the ITU Kaleidoscope 2016. Moreover, he has served as TPC member of more than ten international conferences, including the ITU Kaleidoscope, IEEE VTC, IEEE CCNC, IEEE WCNC, etc. He has been a Lead Guest Editor for Sensors, Peerto-Peer Networking and Applications, Energies and Guest Editor for IEICE Transactions on Information and Systems, Intelligent Automation and Soft Computing, Computer Communications. He is an Editor of the IEEE OPEN JOURNAL OF VEHICULAR TECHNOLOGY (OJVT).

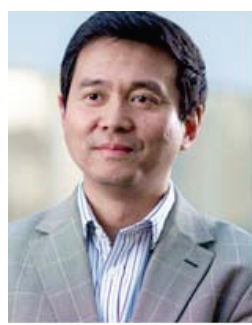

Pei Xiao (Senior Member, IEEE) is a professor of Wireless Communications at the Institute for Communication Systems, home of 5G Innovation Centre (5GIC) at the University of Suring the research team in the new physical layer work area, and coordinating/supervising research activities across all the work areas within 5GIC (www.surrey.ac.uk/5gic/research). Prior to this, he worked at Newcastle University and Queens University Belfast. He also held positions at Nokia Networks in Finland. He has published extensively in the fields of communication theory, RF and antenna design, signal processing for wireless communications, and is an inventor on over 10 recent 5GIC patents addressing bottleneck problems in $5 \mathrm{G}$ systems. rey. $\mathrm{He}$ is the technical manager of $5 \mathrm{GIC}$, lead- 